\title{
On the torsion of isotropic elastoplastic Cosserat circular cylinders
}

\author{
Flavien Ghiglione and Samuel Forest* \\ MINES ParisTech, PSL Research University, MAT - Centre des matériaux, CNRS UMR 7633 \\ BP 8791003 Evry, France
}

\begin{abstract}
Torsional loading of elastoplastic materials leads to size effects which are not captured by classical continuum mechanics and require the use of enriched models. In this work, an analytical solution for the torsion of isotropic perfectly plastic Cosserat cylindrical bars with circular cross-section is derived in the case of generalized von Mises plasticity accounting solely for the symmetric part of the deviatoric stress tensor. The influence of the characteristic length on the microrotation, stress and strain profiles as well as torsional size effects are then investigated. In particular, a size effect proportional to the inverse of the radius of the cylinder is found for the normalized torque. A similar analysis for an extended plasticity criterion accounting for both the couple-stress tensor and the skew-symmetric part of the stress tensor is performed by means of systematic finite element simulations. These numerical experiments predict size effects which are similar to those predicted by the analytical solution. Saturation effects and limit loads are found when the couple stress tensor enters the yield function.
\end{abstract}

Keywords Cosserat, micropolar, elasticity, plasticity, torsion, elastoplasticity, size effect

\section{Introduction}

The theory of Cosserat/micropolar media in the general finite deformation setting has been established by Kafadar and Eringen [1971]; Eringen [1976] and finally reported in the book [Eringen, 1999]. Eringen and Kafadar applied their theory to hyperelasticity of Cosserat media. The extension of this model to finite deformation elasto-visco-plasticity was proposed much later in [Dluzewski, 1992; Sievert, 1995; Sansour, 1998; Forest and Sievert, 2003]. The present work however concentrates on small deformations, rotations and curvatures because this theoretical framework is more easily amenable to analytical solutions in elasticity and even plasticity.

Solutions of many boundary value problems in Cosserat elasticity have been made available in the literature since more than 50 years. Kim and Eringen [1973] solved the problem of the stress concentration at a hole in a micropolar plate. The general Saint-Venant problem was extended to elastic micropolar media in the case of circular cylinders by Reddy and Venkatasubramanian [1976]. The torsion problem is a particular case of the Saint-Venant problem and is the subject of the

\footnotetext{
*Corresponding author: samuel.forest@mines-paristech.fr
} 
present work. Note that the torsion problem has been solved for several other generalized continua like strain gradient elastic media [Lazopoulos and Lazopoulos, 2012; Iesan, 2013; Beheshti, 2018], and recently elastic stress gradient media [Kaiser et al., 2021]. The torsion of Cosserat bars is of physical relevance for the identification of Cosserat elasticity moduli and associated internal length scales [Gauthier and Jashman, 1975]. This test was applied to several materials with highly heterogeneous microstructures like bones [Yang and Lakes, 1981] and other foam materials [Lakes, 1987; Onck, 2002]. Early solutions of the torsion problem for elastic Cosserat bars were provided by Smith [1967]; Ieşan [1971]. In the present work, the solution given by Taliercio and Veber [2016]; Drugan and Lakes [2018] will be recalled and used as a starting point for tackling the elastoplastic case.

Extensions of the von Mises plasticity criterion to Cosserat media go back to pioneering contributions by Neuber [1966, 1968]; Lippmann [1969]; Besdo [1974]; Sawczuk [1967]. First applications to the plasticity of metals were proposed by Diepolder et al. [1991]; Lippmann [1995]. Kaplunov and Lippmann [1995] provided the first asymptotic solution for the elastic-plastic Cosserat continuum in the case of torsion of circular cylinders. More recently, torsion in Cosserat plasticity was considered from the computational perspective by Grammenoudis and Tsakmakis $[2005,2009]$ which allows for the consideration of isotropic and kinematic hardening.

The objective of the present work is to provide a fully explicit analytical solution of the torsion problem for elastic perfectly plastic Cosserat bars with circular crosssection. This is made possible by the use of a simplified version of a general extension of the von Mises yield function to Cosserat media. The reduced form includes an equivalent stress measure that depends solely on the symmetric part of the forcestress tensor. The proposed solution is then compared to finite element results in the case of more general formulations involving the skew-symmetric part of the forcestress tensor and the full couple-stress tensor. The analytical solution is shown to be sufficient to exhibit the main features of the elastic-plastic response of Cosserat bars including stress-strain distributions in the cross-section. In particular it predicts size effects depending on the ratio between the bar radius and the intrinsic length arising in the elasticity solution.

The proposed solution is based on the elastic analysis recalled in section 2 . Section 3 is dedicated to the elastic-plastic case. It contains the formulation of a general extension of the von Mises yield criterion to Cosserat media (section 3.1), the analytical solution in the case of a reduced form of the yield function (section 3.2) and comparison with more general finite element solutions (section 3.4), highlighting the predicted size effects.

\section{Notations}

Vectors and second order tensors are respectively denoted by $\underline{\boldsymbol{a}}$ and $\underset{\sim}{\boldsymbol{A}}$. The transpose is given by $\boldsymbol{\sim}^{T}$. Third and fourth order tensors are respectively written $\underset{\sim}{\boldsymbol{a}}$ and $\underset{\approx}{\boldsymbol{C}}$. Identity tensor of order two and Levi-Civita permutation tensor are called $\underset{\sim}{\tilde{I}}$ and 
$\underset{\epsilon}{\epsilon}$ respectively. The symmetric and skew-symmetric parts of a second order tensor are given by:

$$
{\underset{\sim}{\boldsymbol{s}}}^{\mathrm{sym}}=\frac{1}{2}\left(\underset{\sim}{\boldsymbol{A}}+{\underset{\sim}{\boldsymbol{A}}}^{T}\right), \quad{\underset{\sim}{\boldsymbol{A}}}^{\mathrm{skw}}=\frac{1}{2}\left(\underset{\sim}{\boldsymbol{A}}-{\underset{\sim}{\boldsymbol{A}}}^{T}\right)
$$

The deviatoric part of a second order tensor is written as:

$$
{\underset{\sim}{\boldsymbol{A}^{\prime}}}^{\prime}=\underset{\sim}{\boldsymbol{A}}-\frac{1}{3} \operatorname{tr}(\underset{\sim}{\boldsymbol{A}}) \underset{\sim}{\boldsymbol{I}}
$$

where $\operatorname{trace}(\underset{\sim}{\boldsymbol{A}})=A_{i i}$ is the trace operator. Simple contraction $A_{i j} b_{j}$ is denoted $\underset{\sim}{\boldsymbol{A}} \cdot \underline{\boldsymbol{b}}$ and double contraction $A_{i j} B_{i j}$ is written as $\underset{\sim}{\boldsymbol{A}}: \underset{\sim}{\boldsymbol{B}}$. The Cartesian components of the gradient operator acting on a vector $\underline{\boldsymbol{a}} \otimes \nabla$ are $a_{i, j}=\partial a_{i} / \partial x_{j}$. The components of the divergence operator $\underset{\sim}{\boldsymbol{A}} \cdot \nabla$ acting on a second order tensor are $A_{i j, j}=\partial A_{i j} / \partial x_{j}$.

\section{Torsion of a cylindrical bar in Cosserat linear isotropic elasticity}

\subsection{Problem setting}

Consider a solid cylinder of radius $R$ and height $L$ subjected to an angle of torsion $\pm \vartheta$ at the top and bottom surfaces respectively (figure 1 ). The difference between the angle of torsion at the top and that at the bottom is denoted $\Delta \vartheta$. The angle of torsion per unit length is written as

$$
a=\frac{\Delta \vartheta}{L}
$$

The Cartesian coordinate $\left(\underline{\boldsymbol{e}}_{x}, \underline{\boldsymbol{e}}_{y}, \underline{\boldsymbol{e}}_{z}\right)$ and the cylindrical coordinate $\left(\underline{\boldsymbol{e}}_{r}, \underline{\boldsymbol{e}}_{\theta}, \underline{\boldsymbol{e}}_{z}\right)$ systems are shown in figure 1 . The lateral sides of the cylinder are traction-free, namely:

$$
\underline{\boldsymbol{\sigma}}(r=R) \cdot \underline{\boldsymbol{e}}_{r}=\underline{\mathbf{0}}, \quad \underset{\sim}{\boldsymbol{m}}(r=R) \cdot \underline{\boldsymbol{e}}_{r}=\underline{\mathbf{0}}
$$

where $\boldsymbol{\sigma}$ and $\underset{\sim}{\boldsymbol{m}}$ are the (generally non-symmetric) Cosserat force and couple stress tensors.

\subsection{Governing equations}

The constitutive equations of Cosserat materials are briefly recalled here. The kinematic fields are the displacement and micro-rotation vectors $\underline{u}$ and $\underline{\varphi}$. The strain measures are the strain and curvature tensors $\underset{\sim}{\boldsymbol{e}}$ and $\underset{\sim}{\boldsymbol{\kappa}}$, defined as:

$$
\begin{gathered}
\underset{\sim}{e}=\underline{\boldsymbol{u}} \otimes \nabla+\underset{\underline{\epsilon}}{\underline{\varphi}} \underline{\varphi} \\
\underset{\sim}{\boldsymbol{\kappa}}=\underline{\varphi} \otimes \nabla
\end{gathered}
$$

The work-conjugate variables of the strain measures respectively are the force stress and couple-stress tensors, which, for an isotropic linear elastic medium, are given by:

$$
\underset{\sim}{\boldsymbol{\sigma}}=\lambda \operatorname{trace}(\underset{\sim}{\boldsymbol{e}}) \underset{\sim}{\boldsymbol{I}}+2 \mu \boldsymbol{\sim}^{\mathrm{sym}}+2 \mu_{c} \boldsymbol{e}^{\mathrm{skw}}
$$




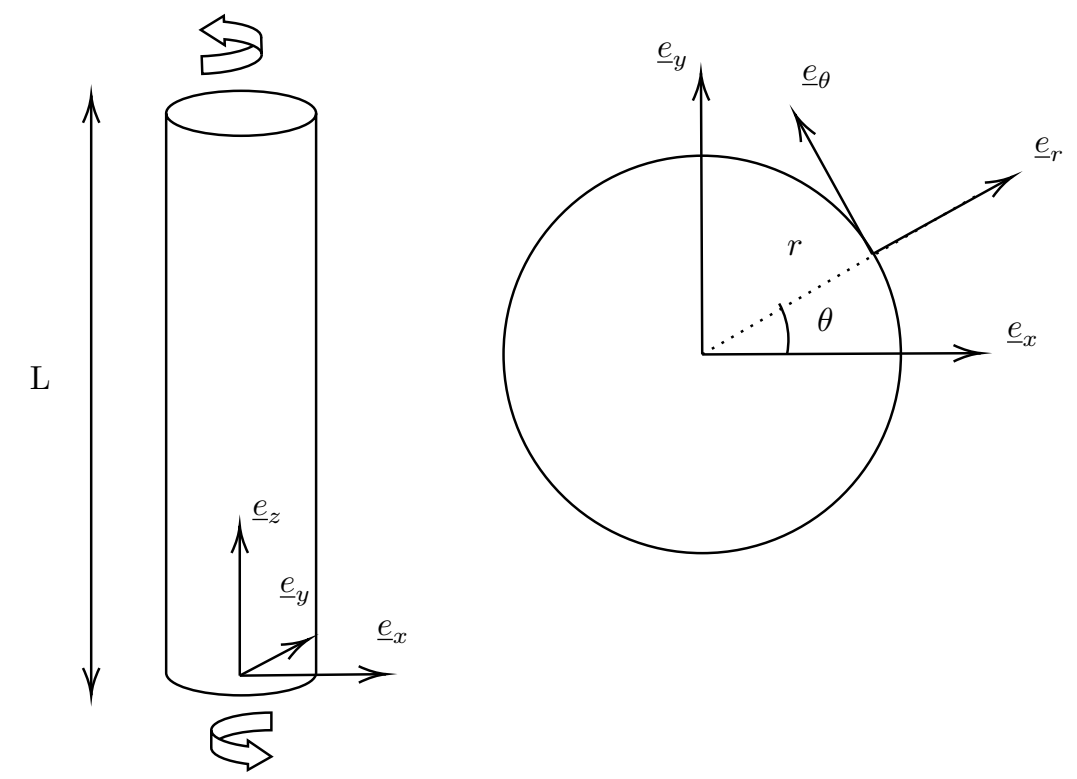

Fig. 1: Torsion of a cylindrical bar and coordinates systems.

$$
\boldsymbol{\sim} \boldsymbol{\sim}=\alpha \operatorname{tr}(\underset{\sim}{\boldsymbol{\kappa}}) \underset{\sim}{\boldsymbol{I}}+2 \beta{\underset{\sim}{\boldsymbol{\kappa}}}^{\mathrm{sym}}+2 \gamma{\underset{\sim}{\boldsymbol{\kappa}}}^{\mathrm{skw}}
$$

where $\lambda$ and $\mu$ are the classical Lamé coefficients, $\mu_{c}$ is the Cosserat coupling modulus and $\alpha, \beta, \gamma$ are Cosserat bending-torsion moduli with force units.

The stress tensors must fulfill the balance of momentum and moment of momentum equations:

$$
\left\{\begin{array}{l}
\underset{\boldsymbol{\sigma}}{\boldsymbol{\sigma}} \cdot \nabla=\underline{\mathbf{0}} \\
\underset{\sim}{\boldsymbol{m}} \cdot \nabla-\underset{\underline{\epsilon}}{\boldsymbol{\epsilon}}: \underset{\sim}{\boldsymbol{\sigma}}=\underline{\mathbf{0}}
\end{array}\right.
$$

here written in the static case in the absence of bulk forces or couples for the sake of brevity. These field equations must be accompanied by boundary conditions, for instance in the form of Neumann conditions:

$$
\underline{\boldsymbol{t}}=\boldsymbol{\sigma} \cdot \underline{\boldsymbol{n}}, \quad \underline{\boldsymbol{m}}=\underset{\sim}{\boldsymbol{m}} \cdot \underline{\boldsymbol{n}}
$$

where $\underline{\boldsymbol{n}}$ is the unit normal vector at any boundary point and $\underline{\boldsymbol{t}}, \underline{\boldsymbol{m}}$ respectively are the traction and couple stress vectors representing applied surface densities of forces and couples. Note that the convention for the definition of the stress tensors is 
the same as in [Toupin, 1962; Borst, 1993]. In many other textbooks like [Nowacki, 1986; Eringen, 1999], the divergence operator and the normal vectors are applied to the left instead of the right side of the second order tensor. This means that the corresponding force and couple stress tensors must be transposed following the latter convention.

\subsection{Problem solution}

The analytical solution for an isotropic elastic Cosserat bar is recalled here in cylindrical coordinates. The displacement and micro-rotation fields are of the form:

$$
\underline{\boldsymbol{u}}=\left(\begin{array}{c}
0 \\
a r z \\
0
\end{array}\right), \quad \underline{\boldsymbol{\varphi}}=\left(\begin{array}{c}
\phi(r) \\
0 \\
a z
\end{array}\right)
$$

The displacement vector is exactly the same as for a classical continuum. The microrotation field keeps the classical component $\varphi_{z}=a z$. However, the $r$ component of the rotation is the unknown function $\varphi_{r}=\phi(r)$ which, for symmetry reasons, can be assumed to only depend on $r$. The strain and curvature tensors are calculated using equations (5) and (6) respectively:

$$
\underset{\sim}{e}=\left(\begin{array}{ccc}
0 & 0 & 0 \\
0 & 0 & a r+\phi \\
0 & -\phi & 0
\end{array}\right), \quad \underset{\sim}{\kappa}=\left(\begin{array}{ccc}
\phi^{\prime} & 0 & 0 \\
0 & \frac{\phi}{r} & 0 \\
0 & 0 & a
\end{array}\right)
$$

where ' denotes a derivation with respect to $r$. The stress and couple-stress tensors then follow from equations (7) and (8):

$$
\begin{gathered}
\boldsymbol{\sigma}=\left(\begin{array}{llc}
0 & 0 & 0 \\
0 & 0 & \left(\mu+\mu_{c}\right) a r+2 \mu_{c} \phi \\
0\left(\mu-\mu_{c}\right) a r-2 \mu_{c} \phi & 0
\end{array}\right) \\
\underset{\sim}{\boldsymbol{m}}=\left(\begin{array}{ccc}
(\alpha+2 \beta) \phi^{\prime}+\alpha\left(\frac{\phi}{r}+a\right) & 0 & 0 \\
0 & (\alpha+2 \beta) \frac{\phi}{r}+\alpha\left(\phi^{\prime}+a\right) & 0 \\
0 & 0 & (\alpha+2 \beta) a+\alpha\left(\phi^{\prime}+\frac{\phi}{r}\right)
\end{array}\right)
\end{gathered}
$$

The balance equation (9) gives:

$$
m_{r r}^{\prime}+\frac{m_{r r}-m_{\theta \theta}}{r}-\left(\sigma_{\theta z}-\sigma_{z \theta}\right)=0
$$

Replacing the force stresses by their expression one gets:

$$
m_{r r}^{\prime}+\frac{m_{r r}-m_{\theta \theta}}{r}-2 \mu_{c}(a r+2 \phi)=0
$$


which can finally be expressed as an ODE for the unknown function $\phi(r)$ :

$$
\phi^{\prime \prime}+\frac{\phi^{\prime}}{r}-\left(\frac{4 \mu_{c}}{\alpha+2 \beta}+\frac{1}{r^{2}}\right) \phi=2 \frac{\mu_{c}}{\alpha+2 \beta} a r
$$

This differential equation is a modified Bessel equation with right-hand side. The regular solution takes the form:

$$
\phi(r)=A I_{1}(r / \ell)-\frac{a}{2} r, \quad \text { with } \quad \ell=\sqrt{\frac{\alpha+2 \beta}{4 \mu_{c}}}
$$

where $I_{1}$ is the modified Bessel function of the first kind, $A$ is an integration constant and $\ell$ is the characteristic length, which only depends of the new moduli introduced in the Cosserat formulation. Note that the term $-\frac{a}{2} r$ is exactly the $r$ component of the material rotation of a classical continuum subjected to torsion. The integration constant $A$ can be determined from the traction-free boundary condition on the lateral sides of the cylinder:

$$
\underset{\sim}{\boldsymbol{m}}(r=R) \cdot \underline{\boldsymbol{e}}_{r}=\underline{\mathbf{0}} \Longrightarrow m_{r r}(r=R)=0 \Longrightarrow A=\frac{\beta a R}{4 \mu_{c} \ell R I_{0}(R / \ell)-2 \beta I_{1}(R / \ell)}
$$

The final expression for $\phi$ finally is:

$$
\phi(r)=\frac{\beta a R}{4 \mu_{c} \ell R I_{0}(R / \ell)-2 \beta I_{1}(R / \ell)} I_{1}(r / \ell)-\frac{a}{2} r
$$

This analytical solution is now illustrated and used to validate the Finite Element implementation of the Cosserat model in Zset software [Besson and Foerch, 1997]. The radius and height of the cylinders are respectively $R=1 \mathrm{~mm}$ and $L=10 \mathrm{~mm}$. The chosen material parameters are given in table 1 , with for all cases $E=70000 \mathrm{MPa}, \nu=0.3$, and $\mu_{c}=50000 \mathrm{MPa}$. The Finite Element mesh of the bar used throughout the paper contains $240003 \mathrm{D}$ quadratic brick elements with reduced integration (8 Gauss points per element) and 102121 nodes. This amounts to more than 600000 degrees of freedom since each node is endowed with 3 translation and 3 microrotation degrees of freedom. The implementation of the Cosserat element in the Zset code is explained in the reference [Forest et al., 2000].

The simplification $\gamma=\beta$ is used throughout. The characteristic length of the medium will be varied in the analysis.

\begin{tabular}{|c|c|c|}
\hline$\ell[\mathrm{mm}]$ & $\alpha[\mathrm{N}]$ & $\beta[\mathrm{N}]$ \\
\hline 0.05 & 300 & 100 \\
\hline 0.1 & 1000 & 500 \\
\hline 0.2 & 4000 & 2000 \\
\hline 0.9 & 62000 & 50000 \\
\hline
\end{tabular}

Table 1: Material parameters used for the calculations. 
Figure 2 shows the influence of $\ell$ on the profiles of $\phi$ normalized by $a R$. Note that in classical Cauchy elasticity, the material rotation along axis $\underline{e}_{r}$ is $-a r / 2$. It can be seen that as $\ell$ increases, so does $\phi$. For the case $\ell \rightarrow 0$ the classical solution is retrieved.

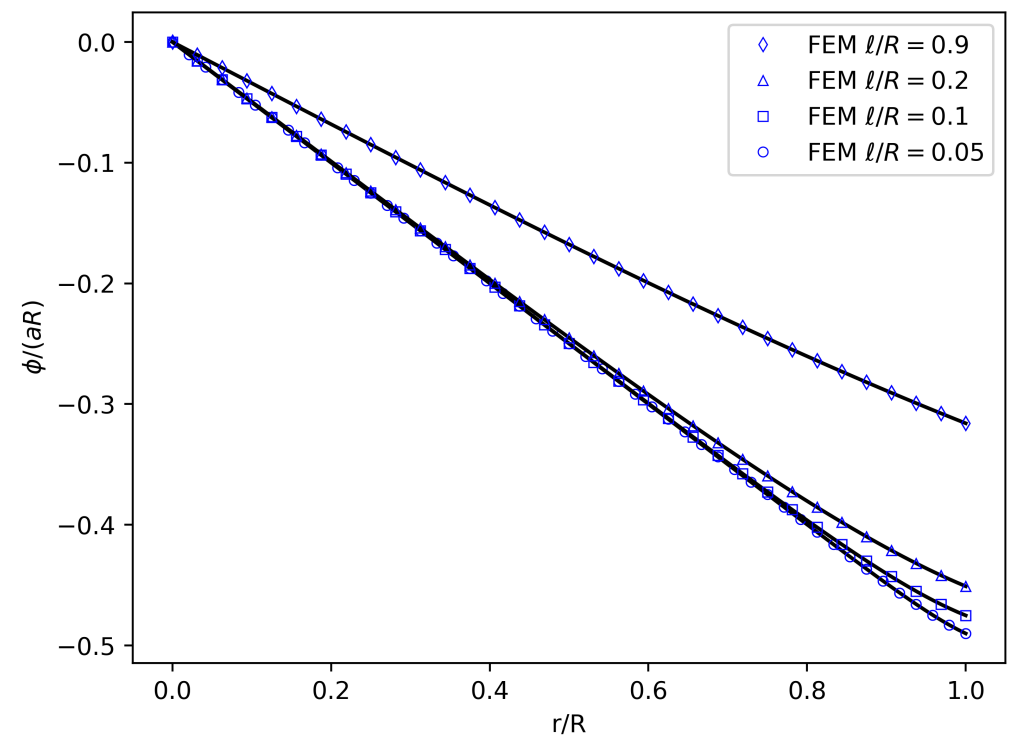

Fig. 2: Profiles of $\phi$ along a radius for various internal lengths for torsion in linear isotropic Cosserat elasticity. Analytical solutions are in black solid lines. Symbols denote Finite Element predictions.

Figure 3 shows the influence of $\ell$ on the stress profiles of $\sigma_{\theta z}$ and $\sigma_{z \theta}$, normalized by the shear at the outer radius for a classical continuum, i.e. $\mu a R$. The curves are roughly symmetric with respect to the line $y=r / R, \sigma_{\theta z}$ and $\sigma_{z \theta}$ being respectively above and below that line. They tend to coincide with that line for decreasing length scale $\ell \rightarrow 0$, where the stress tensor becomes symmetric as expected in the classical case. Strong asymmetry is observed for large values of $\ell$.

Finally, the sensitivity of the components of the couple-stress tensor to the characteristic length is illustrated in figure 4 . The components $m_{r r}, m_{\theta \theta}$ and $m_{z z}$ are normalized by the classical torque divided by the area of a section of the cylinder. The components of the couple-stress tensor are fairly uniform for low internal lengths. An increase in $\ell$ leads to nonlinear profiles as well as an increase of the maximum absolute value attained by the solution. Decreasing $\ell$ to zero leads to vanishing couple stress values, as it should be for a classical continuum. The boundary 


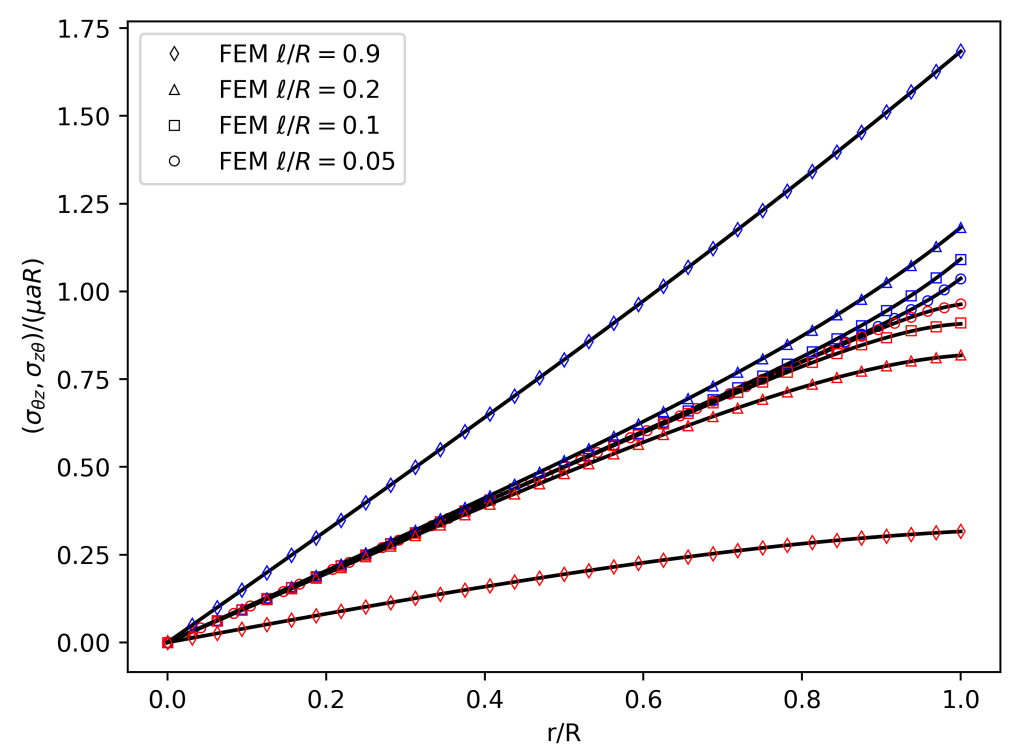

Fig. 3: Profiles of $\sigma_{\theta z}$ (in blue) and $\sigma_{z \theta}$ (in red) along a radius for various internal lengths, for torsion in linear isotropic Cosserat elasticity. Analytical solutions are in black solid lines. Symbols denote Finite Element predictions.

condition $m_{r r}(r=R)=0$ is clearly satisfied, as can be seen from the profile of $m_{r r}$.

\subsection{Size effect}

For a Cosserat continuum, the resulting torque on a given cross-section of the cylindrical bar involves two contributions: One from the stress tensor (moment of forces), as for classical media, and one from the couple-stress tensor (intrinsic surface couples), as follows:

$$
\underline{\boldsymbol{M}}=\int_{0}^{2 \pi} \int_{0}^{R}\left(\underline{\boldsymbol{O M}} \wedge\left(\underline{\sim}_{\sim} \cdot \underline{\boldsymbol{e}}_{z}\right)+\underset{\sim}{\boldsymbol{m}} \cdot \underline{\boldsymbol{e}}_{z}\right) r d r d \theta
$$

The torque $\mathcal{C}$ is then simply the component of $\underline{\boldsymbol{M}}$ with respect to axis $z$, given by:

$$
\mathcal{C}=2 \pi \int_{0}^{R}\left(r \sigma_{\theta z}+m_{z z}\right) r d r d \theta
$$

which in turn gives, in the linear isotropic elastic case:

$$
\mathcal{C}^{e}=\mathcal{C}_{\text {class }}^{e}\left(1+\frac{4 \beta}{\mu R^{2}}\left(1+\frac{\mu_{c} \ell R I_{0}(R / \ell)-\beta I_{1}(R / \ell)}{2 \mu_{c} \ell R I_{0}(R / \ell)-\beta I_{1}(R / \ell)}\right)\right)
$$




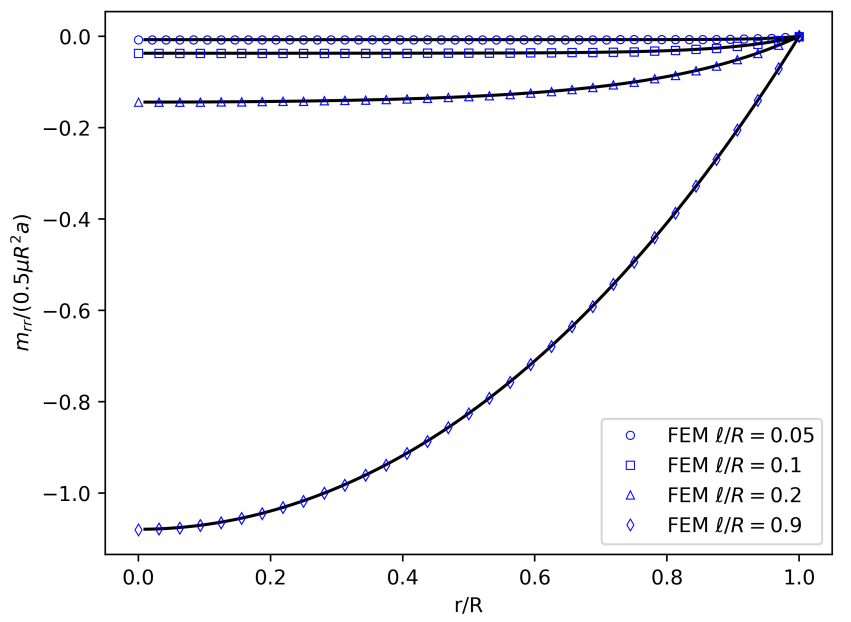

(a) Profiles of $m_{r r}$.

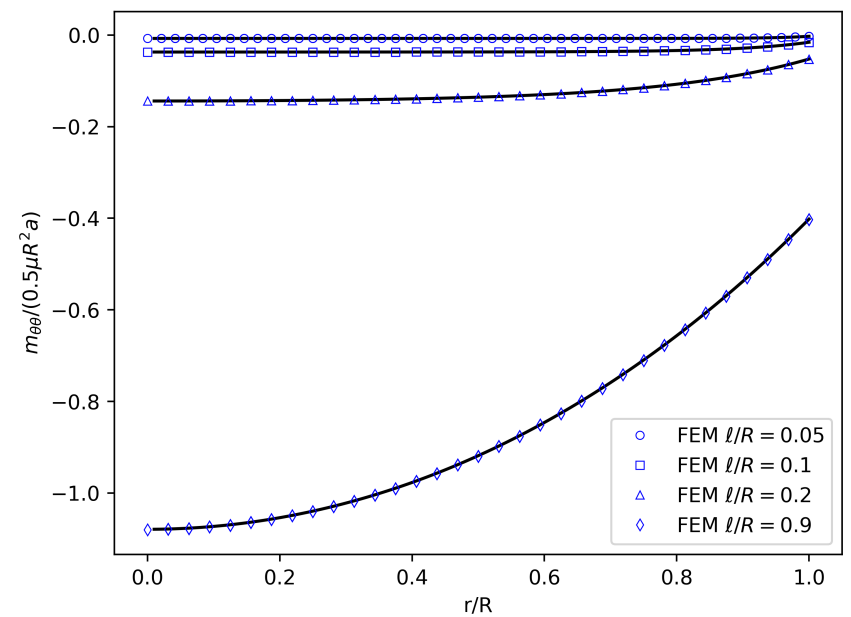

(b) Profiles of $m_{\theta \theta}$.

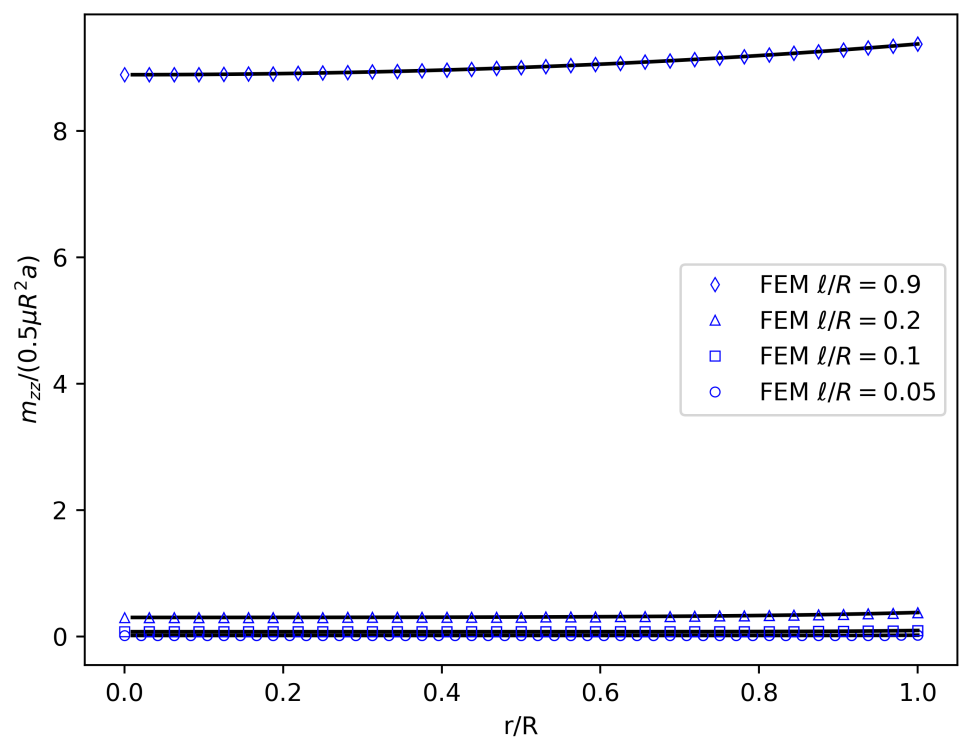

(c) Profiles of $m_{z z}$.

Fig. 4: Profiles of $m_{r r}, m_{\theta \theta}$ and $m_{z z}$ along a radius for various internal lengths in the case of linear isotropic Cosserat elasticity. Analytical solutions are in black solid lines. Symbols denote Finite Element predictions. 
where the torque for a classical continuum reads:

$$
\mathcal{C}_{\text {class }}^{e}=\pi \frac{\mu a R^{4}}{2}
$$

An alternative and useful expression has been derived by Taliercio and Veber [2016]:

$$
\frac{\mathcal{C}^{e}}{\mathcal{C}_{\text {class }}^{e}}=1+\frac{4 \beta}{\mu R^{2}}\left(2-\left(2-\frac{\beta I_{1}(R / \ell)}{\mu_{c} \ell R I_{0}(R / \ell)}\right)^{-1}\right)
$$

Using this expression, various limit cases can be studied. Let us start with the case $R / \ell \rightarrow 0$. For fixed $R$, this limit behavior can be obtained by taking $\ell \rightarrow+\infty$. According to formula (18), this situation can equivalently be studied either by taking $\mu_{c} \rightarrow 0$ keeping other parameter constants (which uncouples the micro-rotation and displacement fields but keeps the contribution of intrinsic couples to the resulting torque) or for both $\alpha, \beta \rightarrow+\infty$.

Using the equivalents

$$
I_{n}(x) \sim \frac{(x / 2)^{n}}{\Gamma(n+1)} \quad \text { for } x \rightarrow 0
$$

one gets:

$$
I_{0}(R / \ell) \underset{\ell \rightarrow+\infty}{\rightarrow} 1, \quad I_{1}(R / \ell) \underset{\ell \rightarrow+\infty}{\rightarrow} \frac{R}{4 \ell}
$$

and finally

$$
\frac{\mathcal{C}^{e}}{\mathcal{\mathcal { C }}_{\text {class }}^{e}} \underset{\ell \rightarrow+\infty}{\rightarrow} 1+\frac{4 \beta}{\mu R^{2}} \frac{3 \alpha+4 \beta}{2 \alpha+3 \beta}
$$

In this expression, a size effect proportional to $R^{-2}$ clearly appears. This can be verified in figure 5 , which illustrates in a log-log plot the ratio of torques as a function of the normalized radius. A fit is also plotted to verify the $R^{-2}$ scaling. It can be seen that as $\frac{R}{\ell} \rightarrow+\infty$ the classical torque is retrieved, whereas for $\frac{R}{\ell} \rightarrow 0$ the ratio of torques increases. Thus, the smaller the radius of the cylinder, the stiffer the response. Note that this result seems to also hold true also for more general prismatic bars, as investigated by Drugan and Lakes [2018].

Another interesting limit case arises when $\mu_{c} \rightarrow+\infty$ and other parameters are fixed, which leads to:

$$
\frac{\mathcal{C}^{e}}{\mathcal{C}_{\text {class }}^{e}} \underset{\ell \rightarrow+0}{\rightarrow} 1+\frac{6 \beta}{\mu R^{2}}
$$

This corresponds to the solution obtained for Koiter's couple stress theory, which constrains the micro-rotation to be equal to the material rotation. 


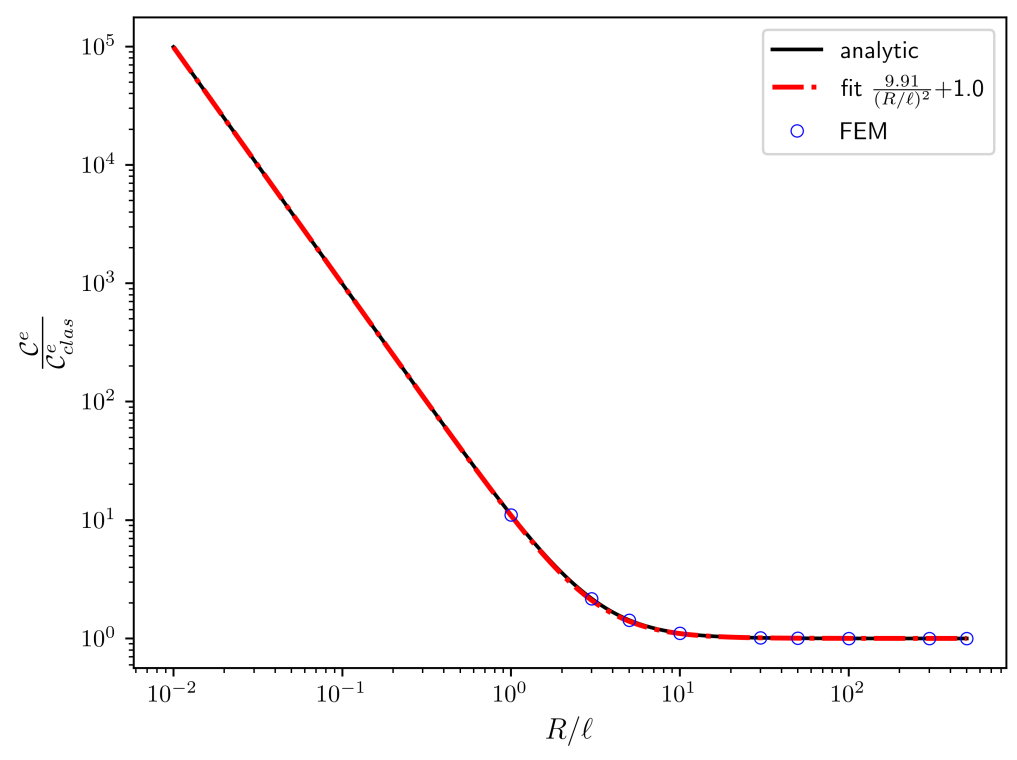

Fig. 5: Cosserat torque normalized by that of a Cauchy continuum as a function of the bar's normalized radius for $\ell=0.1 \mathrm{~mm}$.

\section{Torsion in Cosserat elasto-plasticity}

\subsection{Rate-independent Cosserat plasticity}

The framework of Cosserat rate-independent plasticity in a general case is briefly introduced here. Consider a single, extended von Mises equivalent stress measure incorporating both the stress and couple-stress tensors, adapted from [Besdo, 1974; Diepolder et al., 1991]:

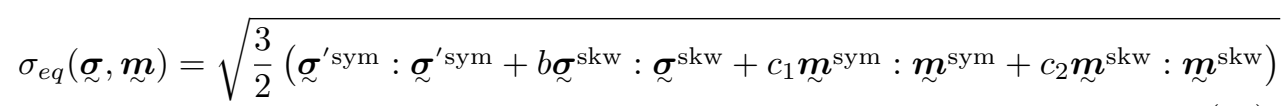

The proposed extended von Mises yield function for the Cosserat continuum is given by:

$$
f(\underset{\sim}{\boldsymbol{\sigma}}, \underset{\sim}{\boldsymbol{m}})=\sigma_{e q}(\underset{\sim}{\boldsymbol{\sigma}}, \underset{\sim}{\boldsymbol{m}})-\sigma_{Y}
$$

where $\sigma_{Y}$ is the current value of the yield stress.

The total deformation and curvature tensors are split into elastic and plastic parts:

$$
\underset{\sim}{e}={\underset{\sim}{e}}^{e}+{\underset{\sim}{e}}^{p}, \underset{\sim}{\boldsymbol{\kappa}}={\underset{\sim}{\boldsymbol{\kappa}}}^{e}+{\underset{\sim}{\boldsymbol{\kappa}}}^{p}
$$


The plastic strain and curvature rates are then derived from a generalized normality rule in the form:

$$
\dot{\sim}^{p}=\dot{p} \frac{\partial f}{\partial \boldsymbol{\sim}}, \quad \dot{\sim}^{p}=\dot{p} \frac{\partial f}{\partial \underset{\sim}{\boldsymbol{m}}}
$$

where $\dot{p}$ is a single plastic multiplier. Time integration of the plastic multiplier provides the path-dependent cumulative plastic strain variable $p$. In the case of the present extended von Mises yield criterion, this gives

$$
\dot{\sim}^{p}=\dot{p} \frac{3}{2} \frac{\underset{\sim}{\boldsymbol{\sigma}^{\prime \mathrm{sym}}+b \boldsymbol{\sim}^{\mathrm{skw}}}}{\sigma_{e q}(\underset{\sim}{\boldsymbol{\sigma}}, \underset{\sim}{\boldsymbol{m}})}, \quad \dot{\sim}^{p}=\dot{p} \frac{3}{2} \frac{c_{1} \boldsymbol{\sim}^{\mathrm{sym}}+c_{2} \boldsymbol{\sim}^{\mathrm{skw}}}{\sigma_{e q}(\boldsymbol{\sigma}, \underset{\sim}{\boldsymbol{m}})}
$$

The plastic multiplier can be expressed as the following norm of the plastic strain and curvature rates:

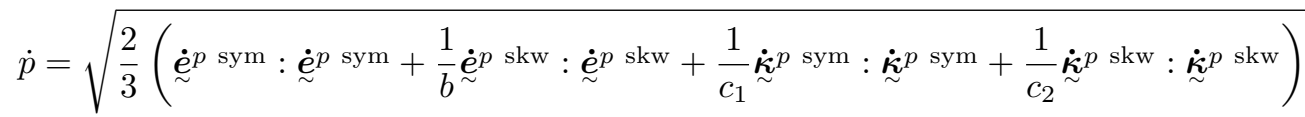

The yield stress can be a function $\sigma_{Y}(p)$ to describe isotropic hardening of the material. In the present work, hardening is not considered for simplicity and $\sigma_{Y}$ is a constant value, the initial yield threshold, corresponding to perfect plasticity.

In the following, the analytical solution is derived in the particular case $b=$ $0, c_{1}=c_{2}=0$ of extended von Mises elastoplasticity in which only the symmetric part of the stress tensor appears in the equivalent stress measure. This analytical solution is compared to Finite Element predictions in more general cases in sections 3.4 and 3.5:

- A criterion accounting for both the symmetric and skew-symmetric parts of the stress tensor in the equivalent stress measure: $b=1, c 1=c 2=0$.

- A criterion accounting for both the symmetric and skew-symmetric parts of the stress tensor as well as the couple-stress tensor in the equivalent stress measure: $b=1, c_{1}=c_{2}=1 \mathrm{~mm}^{-2}$.

\subsection{Analytical solution in a simplified case}

In this section, an analytical solution is derived in the following particular case of formula (30):

$$
b=0, \quad c_{1}=c_{2}=0
$$

The yield function reduces in that case formally to the classical von Mises criterion involving the quadratic norm of the symmetric deviatoric stress tensor:

$$
\sigma_{e q}(\boldsymbol{\sigma})=\sqrt{\frac{3}{2} \boldsymbol{\sigma}^{\prime \mathrm{sym}}: \boldsymbol{\sigma}^{\prime \mathrm{sym}}}
$$

Plasticity occurs when $f(\boldsymbol{\sigma})=0$. In the torsion case, this gives

$$
\frac{3}{4}\left(\sigma_{\theta z}+\sigma_{z \theta}\right)^{2}=\sigma_{Y}^{2}
$$


Making use of equation (38), $\sigma_{\theta z}$ and $\sigma_{z \theta}$ can be related by the following formula:

$$
\sigma_{z \theta}=\frac{2 \sigma_{Y}}{\sqrt{3}}-\sigma_{\theta z}
$$

assuming that the loading is such that $\sigma_{\theta z}+\sigma_{z \theta} \geq 0$.

Starting from the elasticity solution exhibited in the previous section, the yield criterion will be reached at the outer boundary $r=R$ for a critical value of the loading $a_{l}$ computed as

$$
a_{l}=\frac{\sigma_{Y}}{\mu R \sqrt{3}}
$$

For increasing torsion loading, the bar cross-section will be split into a central disc of radius $r_{l}$ where the yield stress has not been reached yet (elastic core), and a ring $r_{l} \leq r \leq R$ in which plastic yielding takes place (plastic zone):

$$
r_{l}=\frac{\sigma_{Y}}{\mu a \sqrt{3}}
$$

For the yield criterion presented in equation (37), the expressions of $r_{l}$ and $a_{l}$ are the same as for a classical continuum.

Figure 6 shows the cumulative plastic strain at two different steps of the torsional loading of the Cosserat elastoplastic bar. The elastic core is shrinking with increasing load.

The total deformation induced by torsion is still given by equation (12). It is now decomposed into elastic and plastic contributions:

$$
\left\{\begin{array}{l}
e_{\theta z}^{e}+e_{\theta z}^{p}=a r+\phi \\
e_{z \theta}^{e}+e_{z \theta}^{p}=-\phi
\end{array}\right.
$$

The elastic strain tensor is obtained by inverting the isotropic elasticity law, equation (7). For monotonic proportional loading, the plastic strain tensor is derived directly from equation (34):

$$
\stackrel{e}{\sim}^{e}=\frac{1}{4 \mu \mu_{c}}\left(\left(\mu+\mu_{c}\right) \boldsymbol{\sigma}+\left(\mu_{c}-\mu\right) \boldsymbol{\sigma}^{T}\right), \quad e^{p}=p \frac{3}{4 \sigma_{Y}}\left(\boldsymbol{\sigma}+\boldsymbol{\sigma}^{T}\right)
$$

Substituting the strains given in equation (43) into the system of equations (42) allows to derive an analytical expression for the plastic strain $p$ :

$$
p=\frac{\sigma_{Y}}{\frac{3}{4}\left(\sigma_{\theta z}+\sigma_{z \theta}\right)}\left(-\phi-\frac{1}{4 \mu \mu_{c}}\left(\sigma_{z \theta}\left(\mu+\mu_{c}\right)+\sigma_{\theta z}\left(\mu_{c}-\mu\right)\right)\right)
$$

Finally, substituting this expression of $p$ in the system of equations (42) and using equation (38), the stress components are fully determined as

$$
\left\{\begin{array}{l}
\sigma_{\theta z}=\mu_{c}(a r+2 \phi)+\frac{\sigma_{Y}}{\sqrt{3}} \\
\sigma_{z \theta}=-\mu_{c}(a r+2 \phi)+\frac{\sigma_{Y}}{\sqrt{3}}
\end{array}\right.
$$

Contrary to the classical case, the previous stress components are not uniform in the plastic zone, even though no hardening is present. Moreover, this stress tensor 
is not symmetric.

Using equation (45), equation (44) simply reduces to the following expression, which is the same as for a Cauchy continuum:

$$
p=\left\{\begin{array}{l}
0 \quad \text { for } r \leq r_{l} \\
\frac{a}{\sqrt{3}}\left(r-r_{l}\right) \quad \text { else }
\end{array}\right.
$$

The profile of $p$ along a normalized radius is shown in figure 7 where it can be seen that $p$ vanishes for $r \leq r_{l}$ and increases linearly for $r>r_{l}$.

The obtained stress tensor must also satisfy the moment of momentum balance equation:

$$
m_{r r}^{\prime}+\frac{m_{r r}-m_{\theta \theta}}{r}-\left(\sigma_{\theta z}-\sigma_{z \theta}\right)=0
$$

Replacing $\sigma_{\theta z}$ and $\sigma_{z \theta}$ by their expression given in equation (45), the balance equation becomes:

$$
m_{r r}^{\prime}+\frac{m_{r r}-m_{\theta \theta}}{r}-\left(\mu_{c}(a r+2 \phi)+\frac{\sigma_{Y}}{2 \sqrt{a_{1}}}-\left(-\mu_{c}(a r+2 \phi)+\frac{\sigma_{Y}}{2 \sqrt{a_{1}}}\right)\right)=0
$$

which reduces to:

$$
m_{r r}^{\prime}+\frac{m_{r r}-m_{\theta \theta}}{r}-2 \mu_{c}(a r+2 \phi)=0
$$

This equation is exactly the same as equation (16) derived in the purely elastic case. Since the simplified criterion (37) does not involve the couple-stress tensor, $\underset{\sim}{\boldsymbol{m}}$ keeps its purely elastic form. The ODE governing $\phi$ therefore is necessarily the same as for the elastic case and given by equation (17). The final expression of $\phi(20)$ is still valid.

The stress fields can be visualized in figure 8. As expected from equation (45) $\sigma_{\theta z}$ has greater values than $\sigma_{z \theta}$. Figure 9 shows the profiles of these stresses normalized by the shear stress value at the outer radius for a classical continuum $\mu a R$, as functions of the normalized radial coordinate. Just like in the elastic case presented in section 2.3 , the stresses symmetrically deviate from the classical solution and the point at which this deviation occurs decreases with increasing internal length. For a large characteristic length the elastic response $\left(r \leq r_{l}\right)$ differs from the Cauchy one and the stress distributions are not symmetric with respect to the classical value any more.

\subsection{Size effect}

As plasticity does not occur for $a \leq a_{l}$, the torque must be separated into two parts: The torque produced for a purely elastic bar and the one corresponding to elastoplastic deformation of the bar. For the elastoplastic part, an elastic core zone exists for $r \leq r_{l}$. The contribution of the stress tensor to the total torque can be 


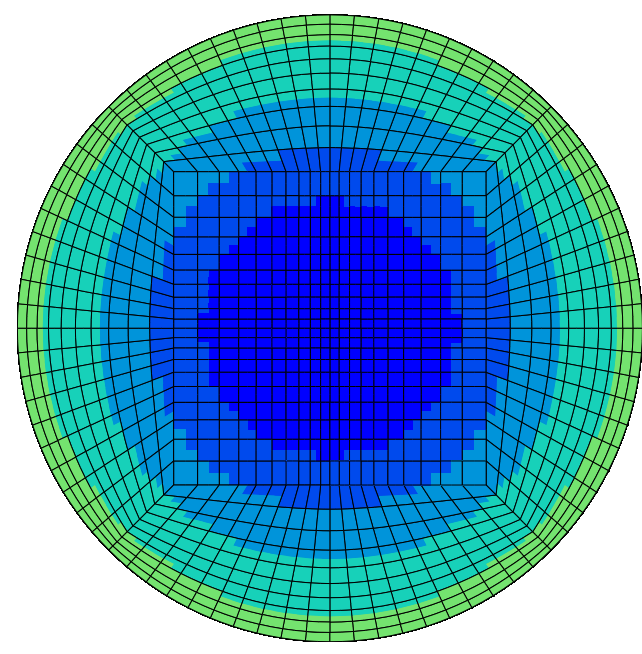

(a) Cumulative plastic strain at $0.44 a_{\max }$

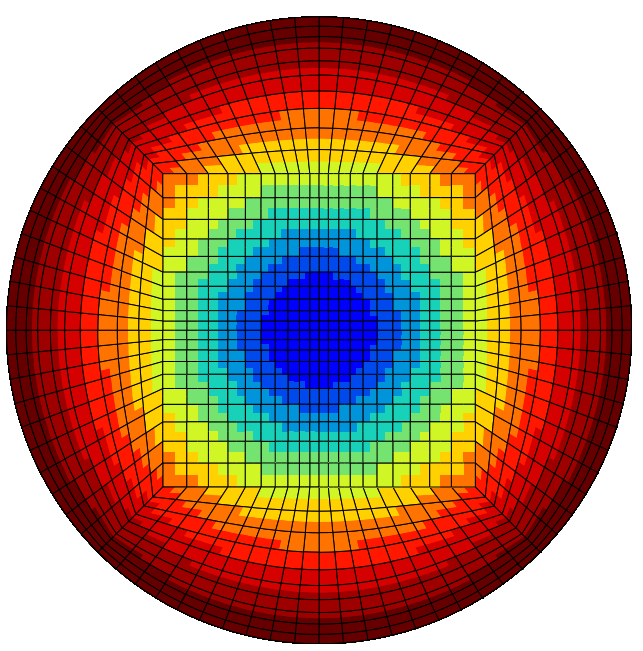

(b) Cumulative plastic strain at $a_{\max }$

Fig. 6: Evolution of the cumulative plastic strain represented on the reference configuration for an imposed maximum angle of torsion $a_{\max }=0.02 \mathrm{rad} / \mathrm{mm}$ and $\ell / R=0.2$. The reduced yield function (37) is used.

split into elastic (for $r \leq r_{l}$ ) and plastic (for $r \geq r_{l}$ ) parts. In the end, the moment takes the following form:

$\mathcal{C}=\left\{\begin{array}{l}2 \pi \int_{0}^{R} r \sigma_{\theta z}^{\text {elastic zone }}+m_{z z} r d r d \theta, \text { for } a \leq a_{l} \\ 2 \pi \int_{0}^{r_{l}} r \sigma_{\theta z}^{\text {elastic zone }} r d r d \theta+\int_{r_{l}}^{R} r \sigma_{\theta z}^{\text {plastic zone }} r d r d \theta+\int_{0}^{R} m_{z z} d r d \theta, \text { for } a \geq a_{l}\end{array}\right.$

After integration, the expressions of the torque are:

$$
\begin{aligned}
\mathcal{C} & =\pi \frac{\mu a R^{4}}{2}\left(1+\frac{4 \beta}{\mu R^{2}}\left(1+\frac{\mu_{c} l R I_{0}(R / \ell)-\beta I_{1}(R / \ell)}{2 \mu_{c} l R I_{0}(R / \ell)-\beta I_{1}(R / \ell)}\right)\right), \quad \text { for } a \leq a_{l} \\
\mathcal{C} & =2 \pi\left[\frac{\mu a r_{l}^{4}}{4}+\frac{\sigma_{Y}}{3 \sqrt{3}}\left(R^{3}-r_{l}^{3}\right)+2 \mu_{c} A \ell^{3}\left(\left(\frac{R}{\ell}\right)^{2} I_{0}(R / \ell)-2 \frac{R}{\ell} I_{1}(R / \ell)\right)\right. \\
& \left.+\beta R^{2} a+\alpha A R I_{1}(R / \ell)\right], \quad \text { for } a \geq a_{l} .
\end{aligned}
$$

The second expression can be expanded by inserting the value (4) of the integration constant $A$ (the same value as in the purely elastic case as discussed earlier), for $a \geq a_{l}$ :

$$
\mathcal{C}=2 \pi\left[\frac{\mu a r_{l}^{4}}{4}+\frac{\sigma_{Y}}{3 \sqrt{3}}\left(R^{3}-r_{l}^{3}\right)+\beta R^{2} a\right.
$$




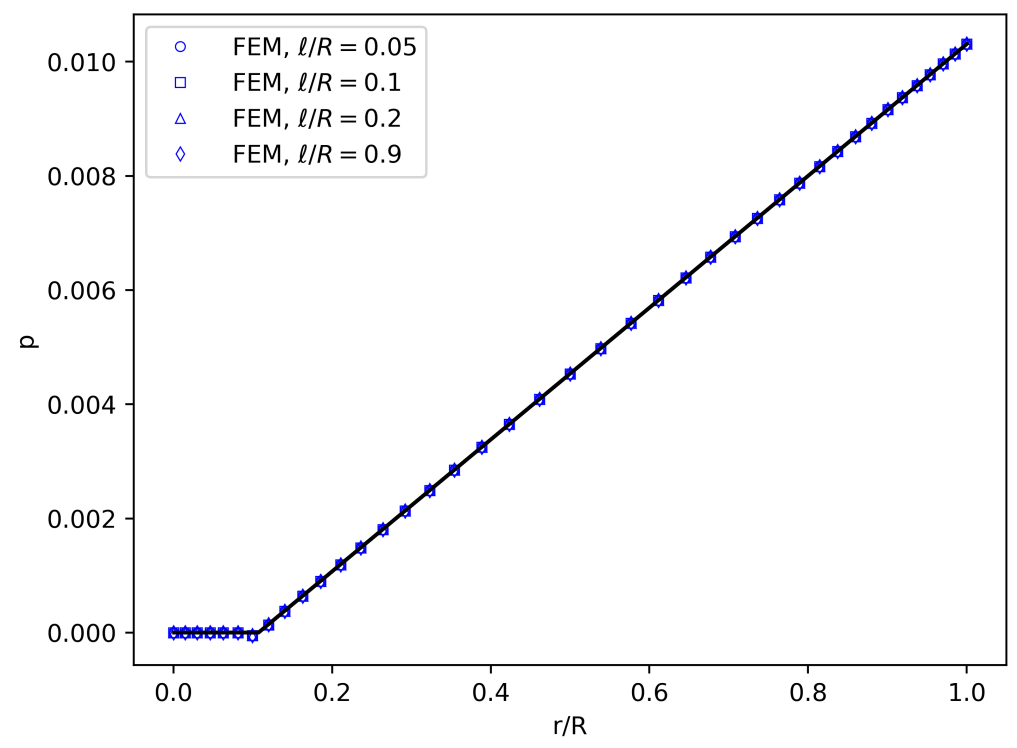

Fig. 7: Profiles of cumulative plastic strain $p$ along a radius for various internal lengths and $a=0.02 \mathrm{rad} / \mathrm{mm}$. Analytical solutions are in black solid lines. Symbols denote Finite Element predictions. The reduced yield function (37) is used.

$$
\left.+\frac{\beta a R}{4 \mu_{c} \ell R I_{0}(R / \ell)-2 \beta I_{1}(R / \ell)}\left(2 \mu_{c} \ell^{3}\left(\left(\frac{R}{\ell}\right)^{2} I_{0}(R / \ell)-2 \frac{R}{\ell} I_{1}(R / \ell)\right)+\alpha R I_{1}(R / \ell)\right)\right]
$$

In the latter expression, the torque for a classical elastoplastic material $\mathcal{C}_{\text {class }}^{p}$ appears:

$$
\mathcal{C}_{\text {class }}^{p}=2 \pi\left[\frac{\mu a r_{l}^{4}}{4}+\frac{\sigma_{Y}}{3 \sqrt{3}}\left(R^{3}-r_{l}^{3}\right)\right]
$$

For a Cauchy continuum the torque-twist angle curve exhibits a plateau as $a \rightarrow \infty$, which corresponds to the case where the plastic zone invades the whole cylinder, $r_{l} \rightarrow 0$. This limit value of the torque is given by:

$$
\mathcal{C}_{\text {class }}^{\infty}=\lim _{a \rightarrow \infty} \mathcal{C}_{\text {class }}^{p}=\frac{2 \pi \sigma_{Y}}{3 \sqrt{3}} R^{3}
$$

However, for a Cosserat continuum with the yield criterion involving the reduced invariant (37), such a saturation of the torque does not occur. This is illustrated in figure 10. This figure shows the total torque as well as its different contributions normalized by $R^{3}$ as functions of the shear strain at the outer radius, as classically 
done in other works [Fleck et al., 1994; Grammenoudis and Tsakmakis, 2009] for both analytical and FEM solutions. Although no hardening is present in the model, an apparent hardening is visible on this curve. This is due to the last term proportional to the twist angle $a$ in the equation (53). This term has two contributions. One is related to the fact that there is no limit for the couple stress component $m_{z z}$ since it does not enter the reduced yield criterion. The second contribution stems from the fact that the sum $\sigma_{\theta z}+\sigma_{z \theta}$ is bounded due to the yield condition, but not the individual components. An excellent agreement is found between the analytical and finite element solutions, as it should.

As for the elastic case, a size effect occurs with the elastoplastic model studied in this section. Consider $\ell \rightarrow+\infty$, combining the equivalents given in equation (27) withe the expression of the torque in the plastic zone equation (53)one gets:

$$
\mathcal{C} \sim \mathcal{C}_{\text {class }}+2 \pi \beta R^{2} a\left(1+\frac{\alpha+\beta}{2 \alpha+3 \beta}\right)
$$

and the expression of the normalized torque is then:

$$
\frac{\mathcal{C}}{\mathcal{C}_{\text {class }}^{\infty}} \sim \frac{\mathcal{C}_{\text {class }}}{\mathcal{C}_{\text {class }}^{\infty}}+\frac{3 \sqrt{3} \beta}{\sigma_{Y}}\left(1+\frac{\alpha+\beta}{2 \alpha+3 \beta}\right) \frac{a}{R}
$$

Agreement between the expression of the torque (53) and its equivalent (57) has been studied numerically and holds true for $\alpha \gg \beta$. Moreover, equation (57) can be further simplified :

$$
\frac{\mathcal{C}}{\mathcal{C}_{\text {class }}^{\infty}} \sim 1+\frac{3 \sqrt{3} \beta}{\sigma_{Y}}\left(1+\frac{\alpha+\beta}{2 \alpha+3 \beta}\right) \frac{a}{R}
$$

From this expression, a size effect clearly appears. In contrast to the elastic case, the normalized torque is proportional to $R^{-1}$, instead of $R^{-2}$, and depends on $a$. This is due to the fact that the normalization $C_{\text {class }}^{\infty}$ is proportional to $R^{3}$ (instead of $R^{4}$ for $\left.C_{\text {class }}^{e}\right)$ and does not depend on $a$, contrary to $C_{\text {class }}^{e}$. This size effect is illustrated in figure 11. These figures show, in the case $a \geq a_{l}$ (i.e. $R \geq \frac{\sigma_{Y}}{\mu a \sqrt{3}}$ ), the torque (53) normalized by the limit torque of a classical continuum as a function of the relative coordinate $R / \ell$, for two different values of $a$. The fitted curves confirm that the size effect is indeed proportional to $R^{-1}$. As mentioned above, the size effect is sensitive to the twist angle $a$, leading to a straighter curve for higher values of $a$. Once again, a good agreement is found between the analytical and FEM solutions.

\subsection{FEM analysis in a more general case: Effect of $\sigma^{\text {skw }}$}

In this section, the influence of the skew-symmetric part of the stress tensor in the yield criterion is numerically investigated. The following equivalent stress measure is considered:

$$
\sigma_{e q}=\sqrt{\frac{3}{2}\left(\boldsymbol{\sigma}^{\prime \mathrm{sym}}: \boldsymbol{\sigma}^{\prime \mathrm{sym}}+\boldsymbol{\sigma}^{\mathrm{skw}}: \boldsymbol{\sigma}^{\mathrm{skw}}\right)}
$$


which is equivalent to:

$$
\sigma_{e q}(\underset{\sigma}{\boldsymbol{\sigma}})=\sqrt{\frac{3}{2}\left(\boldsymbol{\sigma}^{\prime}: \boldsymbol{\sigma}^{\prime}\right)}
$$

This corresponds to the following values of parameters in the general equivalent stress measure (30):

$$
b=1, \quad c_{1}=c_{2}=0
$$

With these parameter values, the yield function is still given by equation (31). The nonlinear constitutive equations are integrated using an implicit Newton $\theta-$ method or by a fourth order Runge-Kutta method with automatic time-stepping [Forest et al., 2000]. The case $\theta=1$ boils down to the classical radial return algorithm in plasticity.

The profiles of $\phi$ normalized by $a R$ along a normalized radius are shown in figure 12 for several internal length values.

The stress profiles are completely different from what was obtained with the reduced criterion (37), as illustrated in figure 13. The values are much lower and for large internal lengths, $\sigma_{z \theta}$ is no longer the symmetric image of $\sigma_{\theta z}$ with respect to the curve obtained for a classical continuum.

Finally, the normalized profiles of $m_{r r}, m_{\theta \theta}$ and $m_{z z}$ are shown in figure 14. Compared to the profiles obtained with criterion (37), the values are lower and the curves are straighter and more spread, although it is not the case for $m_{z z}$.

The normalized torque-twist angle curve is plotted in figure 15 for criterion (60). Contrary to the curve obtained with criterion (37), the contribution due to $\sigma_{\theta z}$ seems to reach a plateau. The one due to $m_{z z}$ keeps increasing as it does not enter the yield criterion, just like with yield function (37). The values are of the same order of magnitude for both yield functions, although those for the contribution due to $m_{z z}$ is a little bit higher.

A size effect is also predicted by criterion (60), as shown in figure 16. The normalized torque is still proportional to $R^{-1}$ and sensitive to the twist angle, just like with criterion (37). 


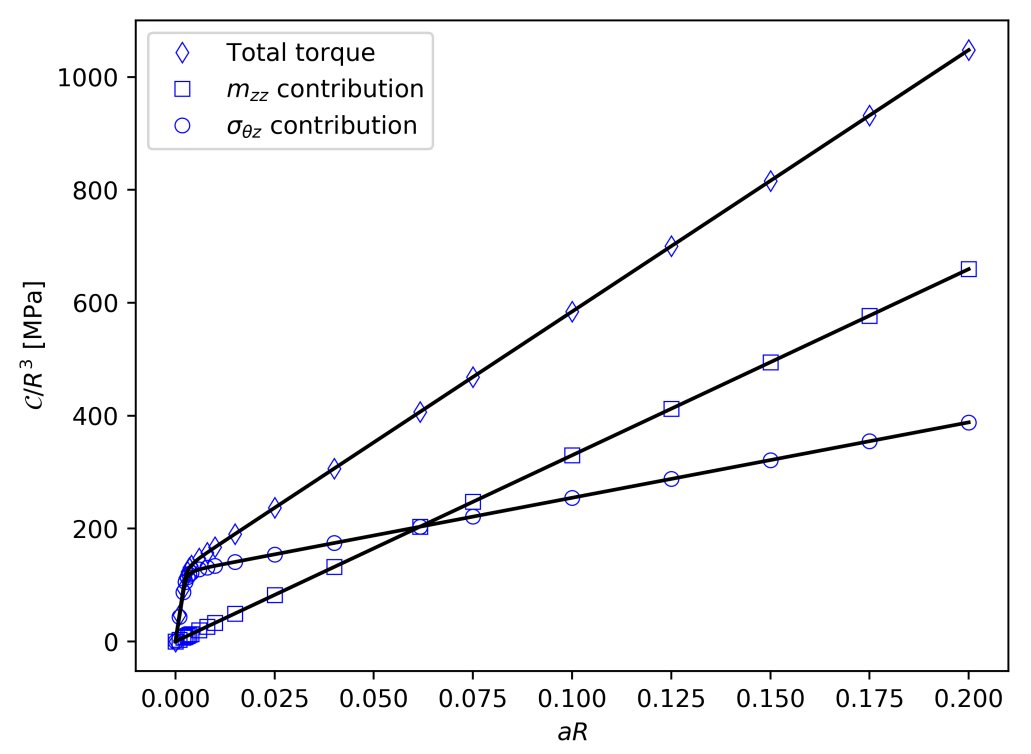

(a) Normalized torque as a function of the classical shear strain.

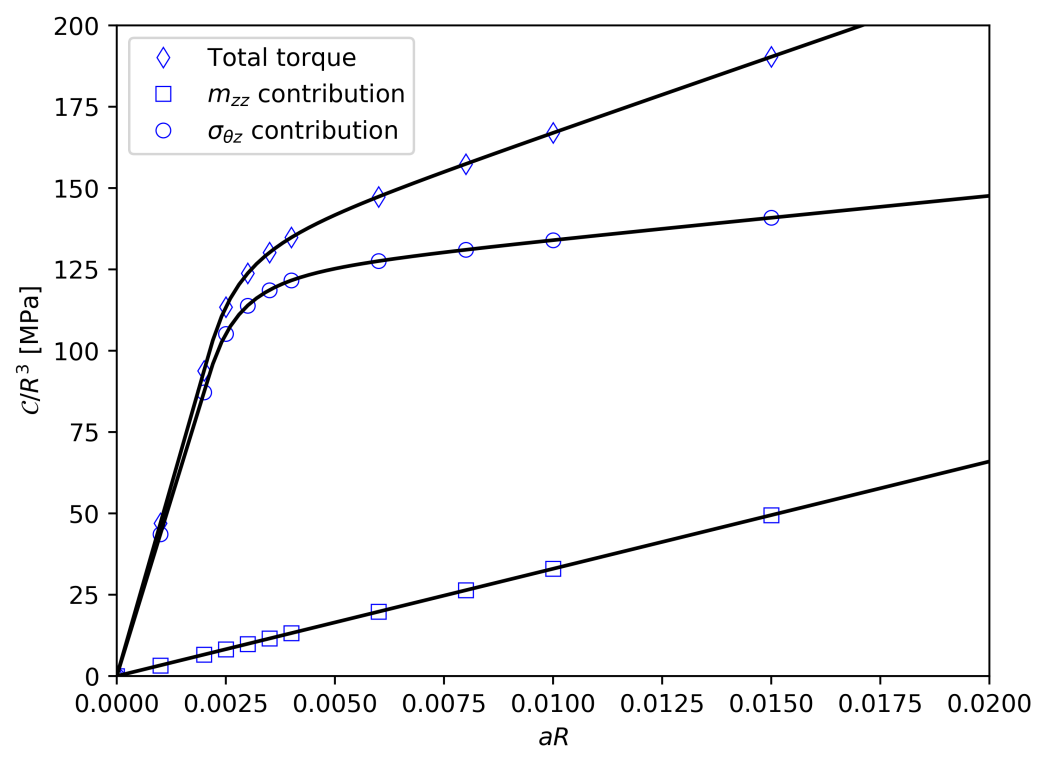

(b) Normalized torque as a function of the classical shear strain, zoom on the beginning of the curve.

Fig. 10: Normalized torque as a function of the classical shear strain with $\ell / R=0.1$.

Analytical solutions are in black solid lines. The reduced equivalent stress (37) is used in the yield function. 


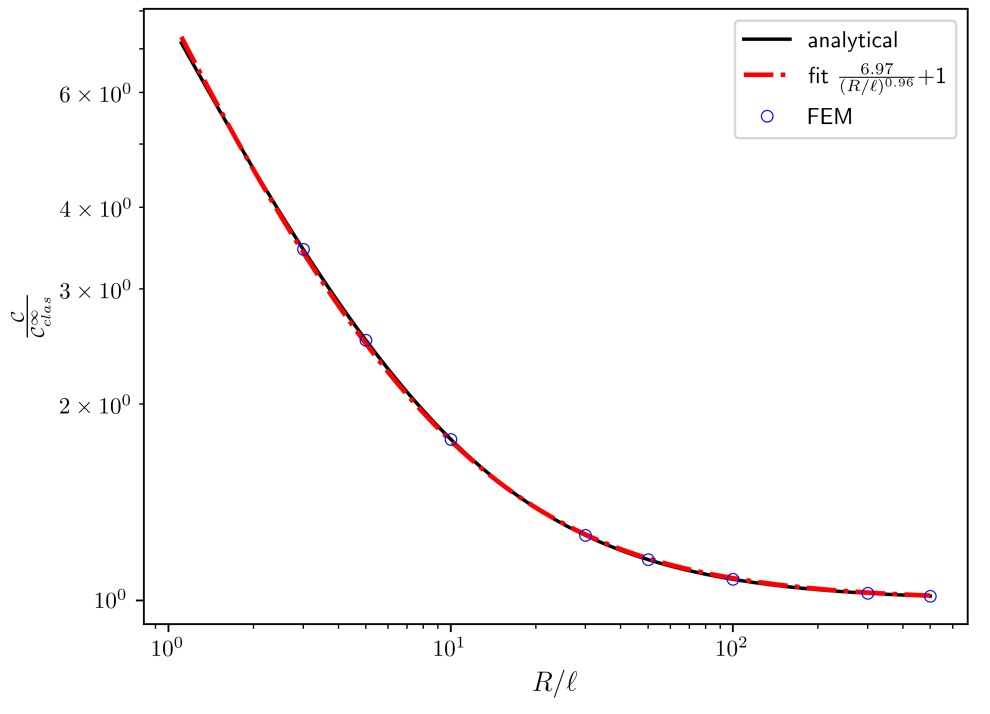

(a) Normalized torque as a function of the normalized radius for $\ell=0.1 \mathrm{~mm}$ and $a=0.02 \mathrm{rad} / \mathrm{mm}$.

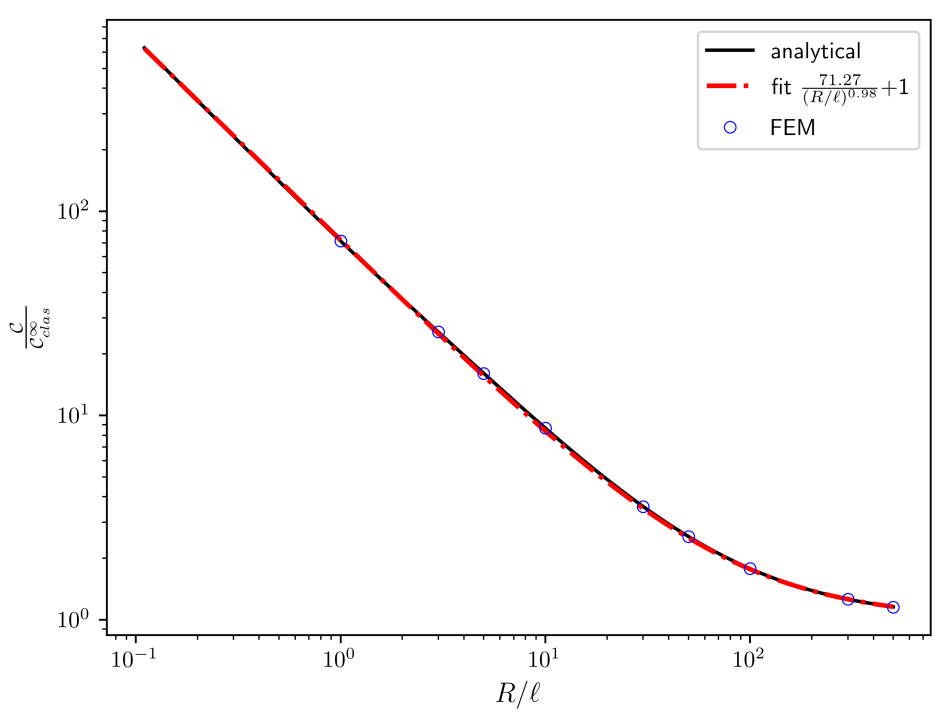

(b) Normalized torque as a function of the normalized radius for $\ell=0.1 \mathrm{~mm}$ and $a=0.2 \mathrm{rad} / \mathrm{mm}$.

Fig. 11: Torque normalized by the limit torque of a classical continuum as a function of the normalized radius for $\ell=0.1 \mathrm{~mm}$ and two values for $a$. Cylinder radius $R$ is such that $R>\frac{\sigma_{Y}}{\mu a \sqrt{3}}$ thus ensuring plastic yielding. The reduced equivalent stress (37) is used in the yield function. 
22 F. Ghiglione and S. Forest

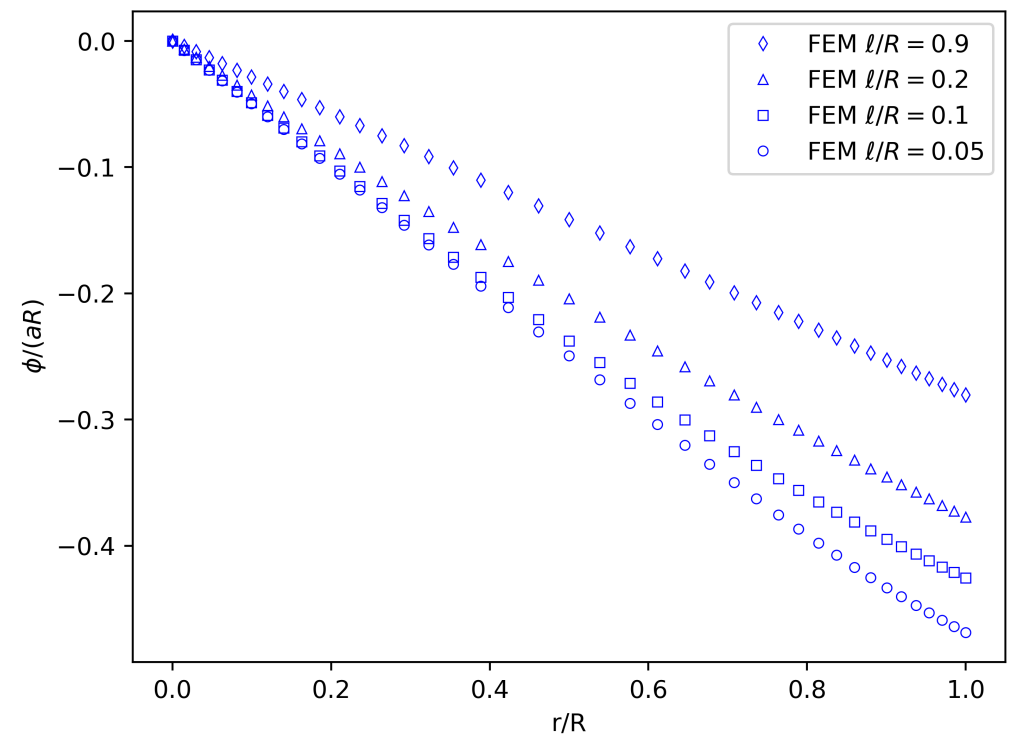

Fig. 12: Profiles of $\phi$ along a radius for various internal lengths and $a=$ $0.02 \mathrm{rad} / \mathrm{mm}$. The equivalent stress used in the yield function is given by equation $(60)$. 


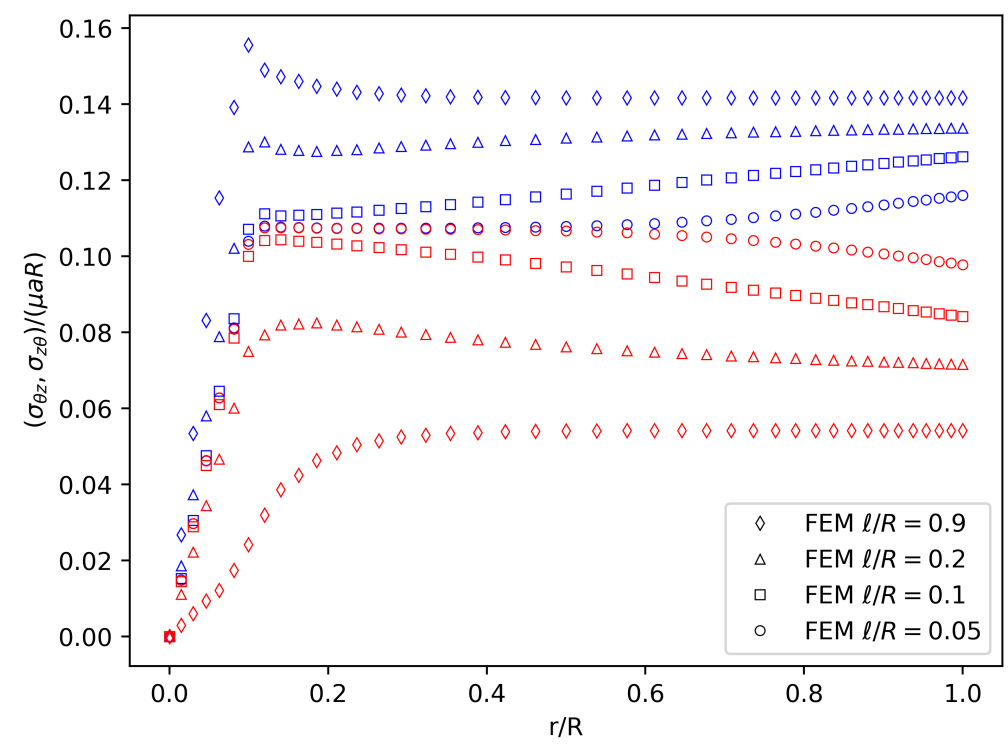

Fig. 13: Profiles of $\sigma_{\theta z}$ (in red) and $\sigma_{z \theta}$ (in blue) along a radius for various internal lengths and $a=0.02 \mathrm{rad} / \mathrm{mm}$. The equivalent stress used in the yield function is given by equation (60). 
24 F. Ghiglione and S. Forest

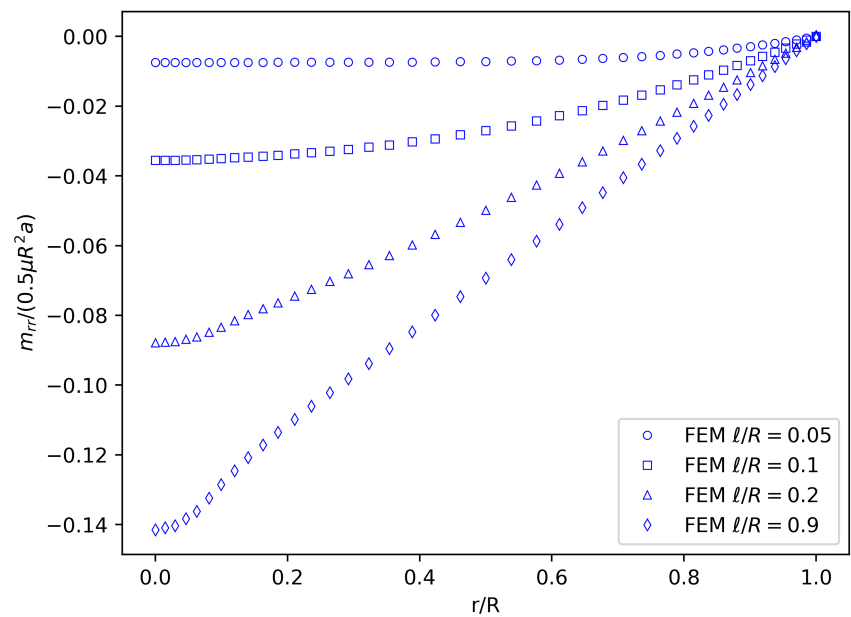

(a) Profiles of $m_{r r}$.

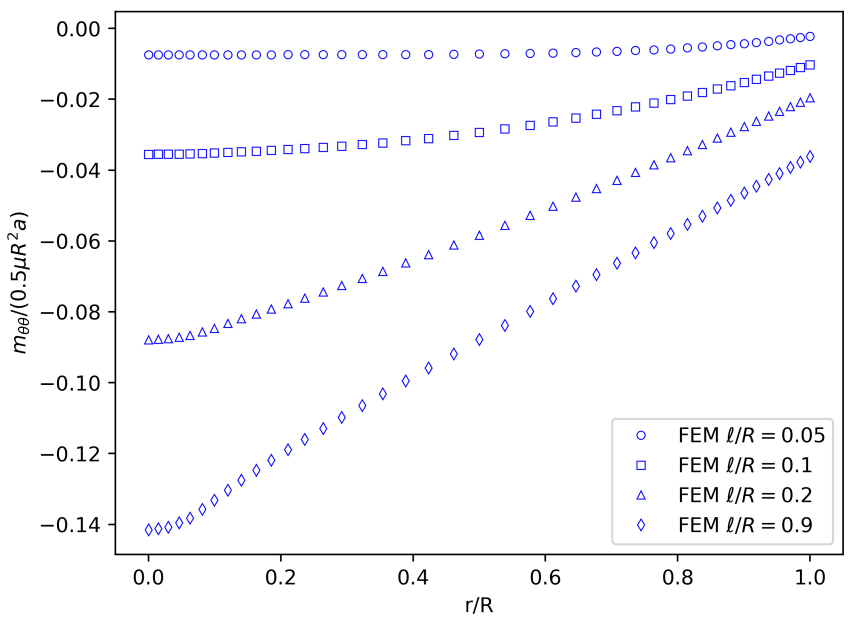

(b) Profiles of $m_{\theta \theta}$.

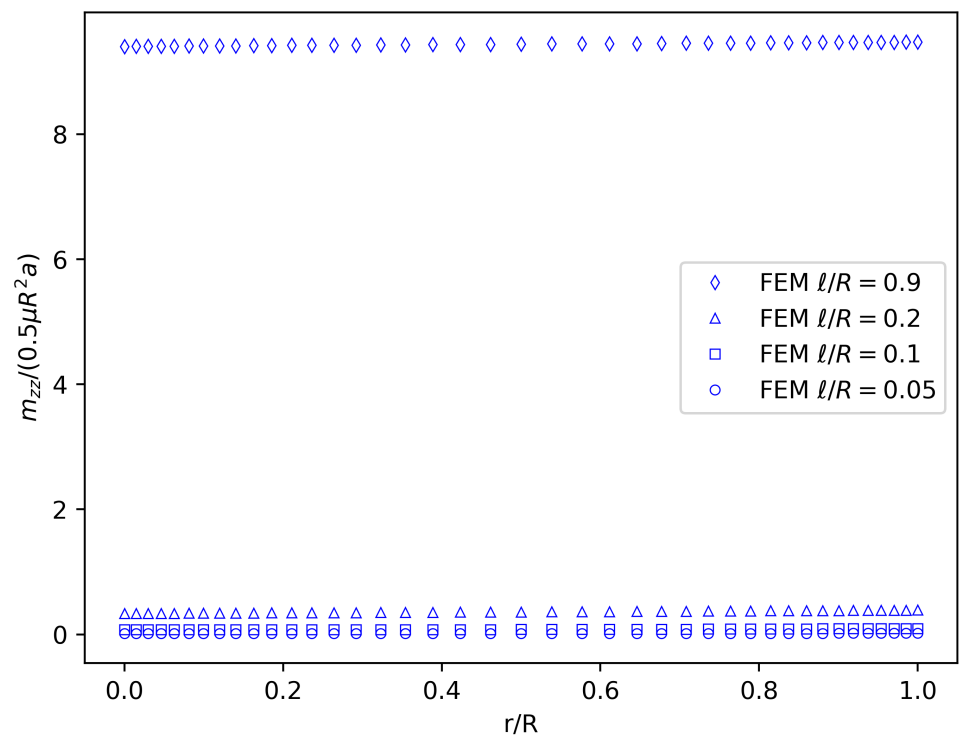

(c) Profiles of $m_{z z}$.

Fig. 14: Profiles of $m_{r r}, m_{\theta \theta}$ and $m_{z z}$ along a radius for various internal lengths and $a=0.02 \mathrm{rad} / \mathrm{mm}$. The equivalent stress used in the yield function is given by equation (60). 


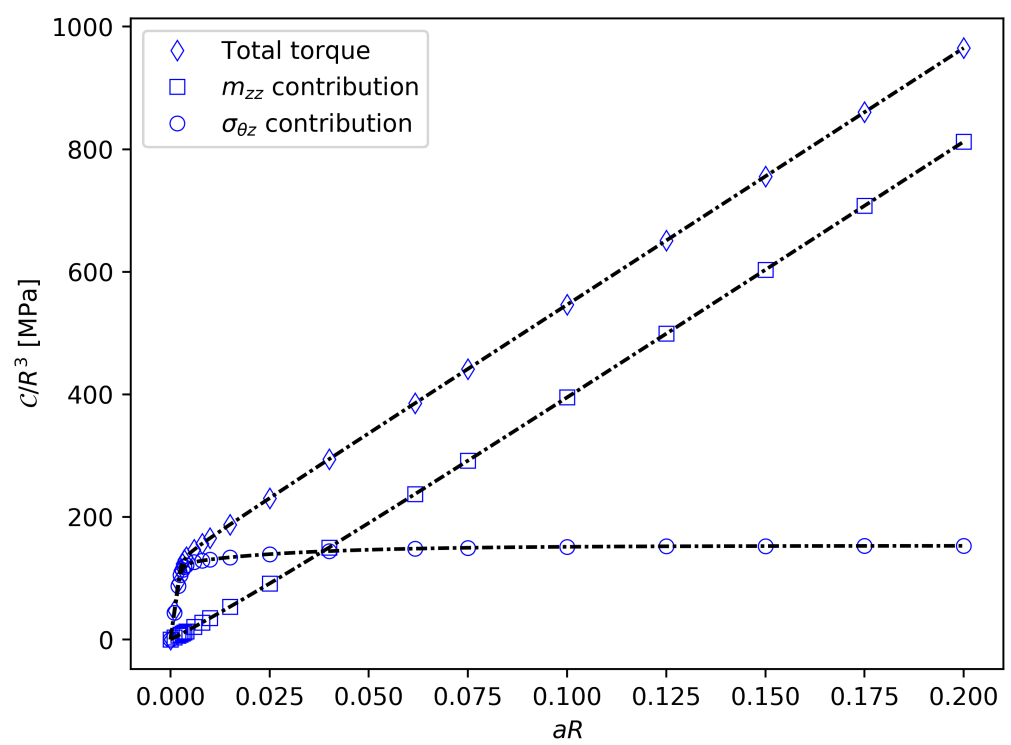

Fig. 15: Normalized torque as a function of the shear with $\ell / R=0.1$. Interpolated solutions are in black dash-dotted lines. The equivalent stress used in the yield function is given by equation (60). 


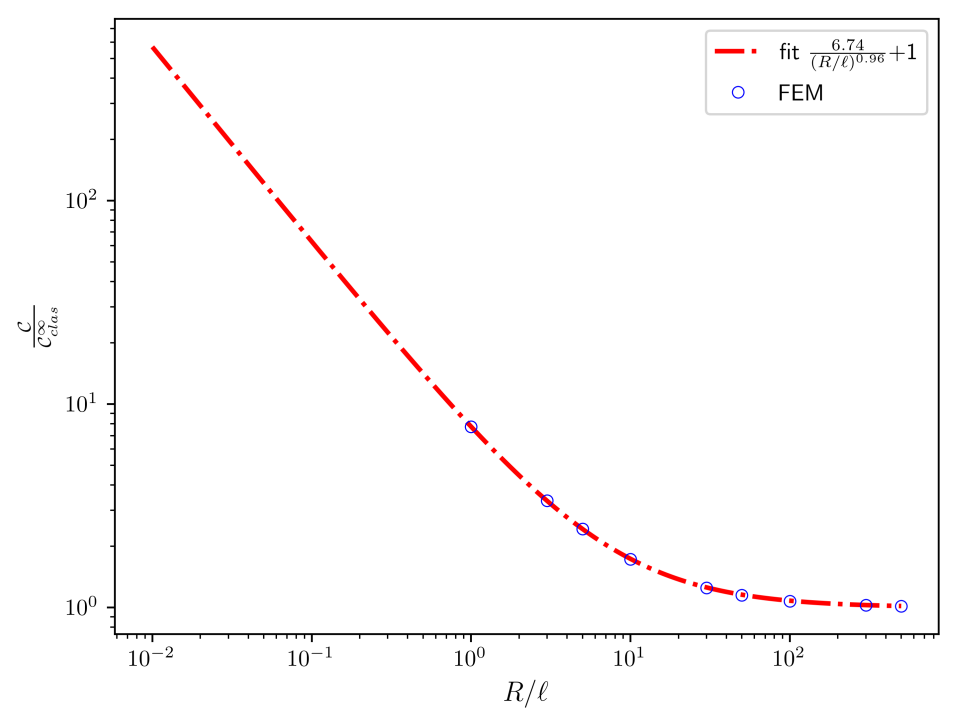

(a) Normalized torque as a function of the normalized radius for $\ell=0.1 \mathrm{~mm}$ and $a=0.02 \mathrm{rad} / \mathrm{mm}$.

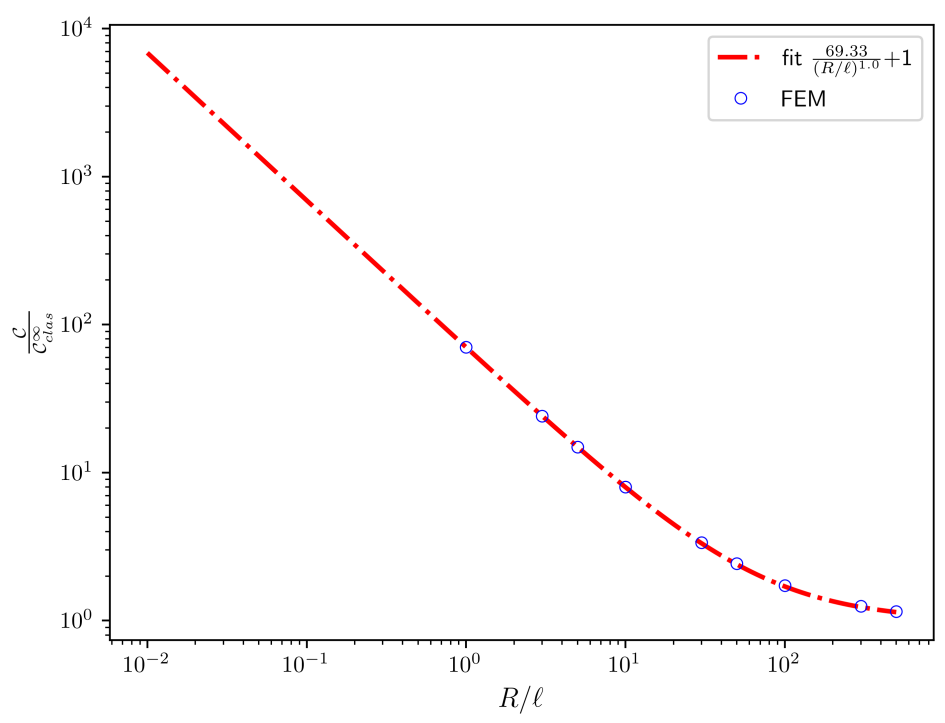

(b) Normalized torque as a function of the normalized radius for $\ell=0.1 \mathrm{~mm}$ and $a=0.2 \mathrm{rad} / \mathrm{mm}$.

Fig. 16: Torque normalized by the limit torque of a classical continuum as a function of the normalized radius for $\ell=0.1 \mathrm{~mm}$ and two values of $a$. The equivalent stress used in the yield function is given by equation (60). 


\subsection{FEM analysis in a more general case: Effect of ${\underset{\sim}{\text { skw }}}^{\text {and } \underset{\sim}{\boldsymbol{m}}}$}

In this section, the yield criterion considered takes into account both the couplestress tensor and the skew-symmetric part of the stress tensor. The chosen equivalent stress measure is taken as

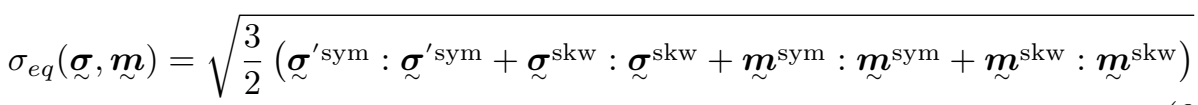

which reduces to:

$$
\sigma_{e q}(\underset{\sim}{\boldsymbol{\sigma}}, \underset{\sim}{\boldsymbol{m}})=\sqrt{\frac{3}{2}\left(\underset{\sim}{\boldsymbol{\sigma}^{\prime}}: \boldsymbol{\sigma}^{\prime}+\underset{\sim}{\boldsymbol{m}}: \underset{\sim}{\boldsymbol{m}}\right)}
$$

This corresponds to the following values of the parameters of the general equivalent stress (30):

$$
b=1, \quad c_{1}=c_{2}=1 \mathrm{~mm}^{-2}
$$

The yield function is still defined by equation (31) using the definition (63) of the equivalent stress.

The normalized profiles of $\phi$ for several internal length values are plotted in figure 17. The results are fairly close to those obtained with yield function (60) except for large length scales, for which the curves are less straight and the range of values is smaller.

As for the stress profiles shown in figure 18, for low characteristic lengths the results are similar to those obtained with yield function (60) in terms of order of magnitude and shape of the curves. However, the behavior is totally different for large internal length values, the linear elastic part of the curves becomes smaller and the stresses become lower.

Figure 19 shows the normalized profiles of the couple stress tensor components. Contrary to the results obtained with the previous yield functions, the yield function (63) predicts a saturation of the size effect: The profiles for $\ell / R=0.2$ and $\ell / R=0.9$ do not differ much. Another difference is that the $m_{z z}$ component is much lower.

The normalized torque-twist angle curves obtained with yield function (63) are shown in figure 20. It can be seen that both contributions from the couple-stress and stress tensors display a saturation. Contrary to the curves obtained using the other criteria, the contribution due to the couple stress is not monotonically increasing and exhibits a decrease after an initial increase, before it reaches a plateau.

Finally, the size effect evidenced for the normalized torque in figure 21 is still proportional to $R^{-1}$ and sensitive to the the twist angle, just like the other yield criteria studied in this work. 


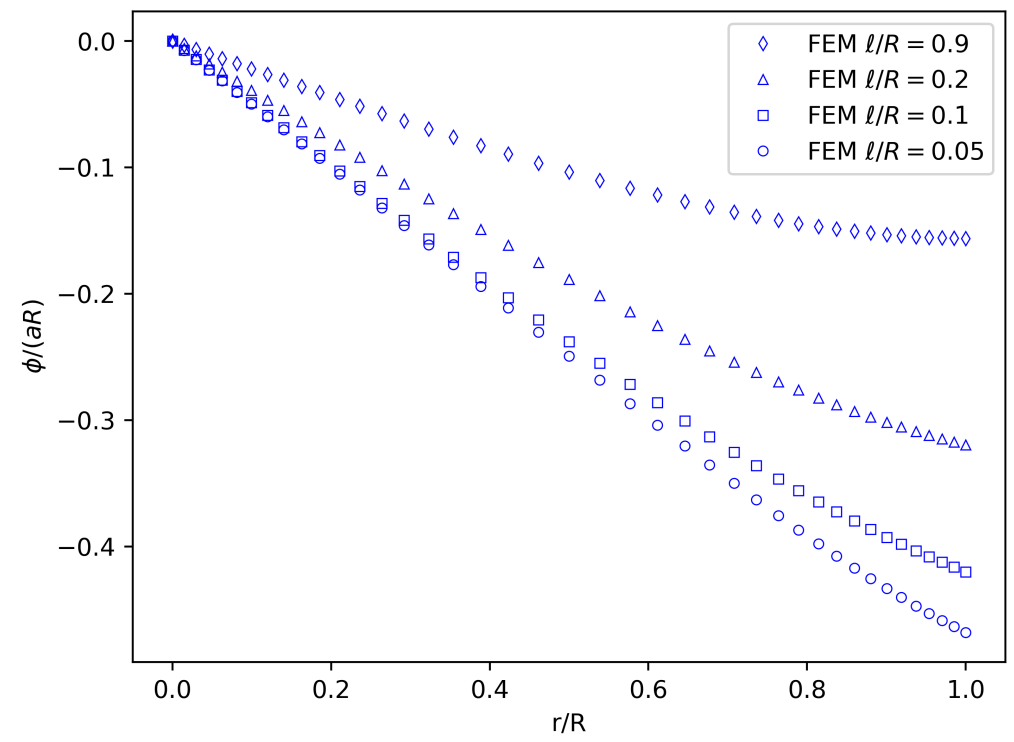

Fig. 17: Profiles of $\phi$ along a radius for several internal length values and $a=$ $0.02 \mathrm{rad} / \mathrm{mm}$. The equivalent stress used in the yield function is given by equation (63).

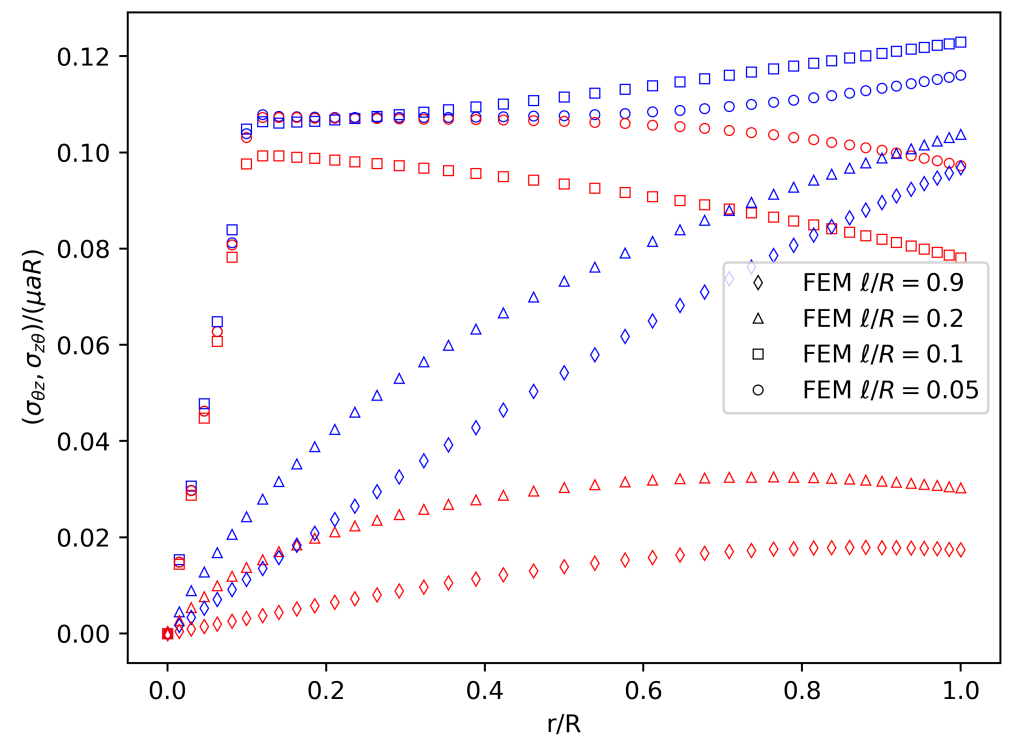

Fig. 18: Profiles of $\sigma_{\theta z}$ (in red) and $\sigma_{z \theta}$ (in blue) along a radius for several internal length values and $a=0.02 \mathrm{rad} / \mathrm{mm}$. The equivalent stress used in the yield function is given by equation (63). 


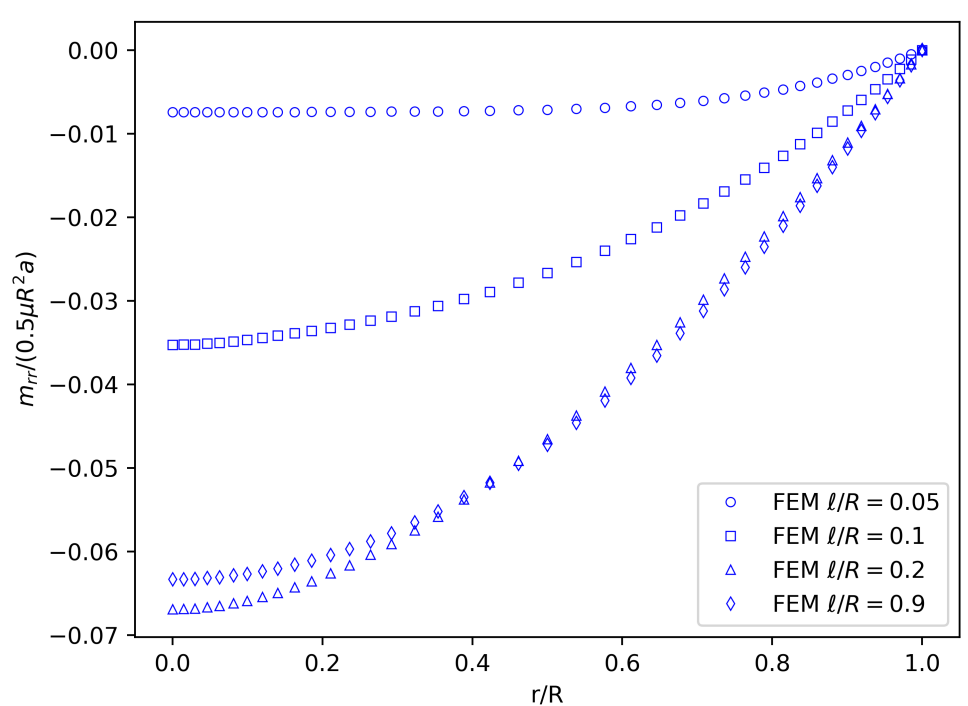

(a) Profiles of $m_{r r}$.

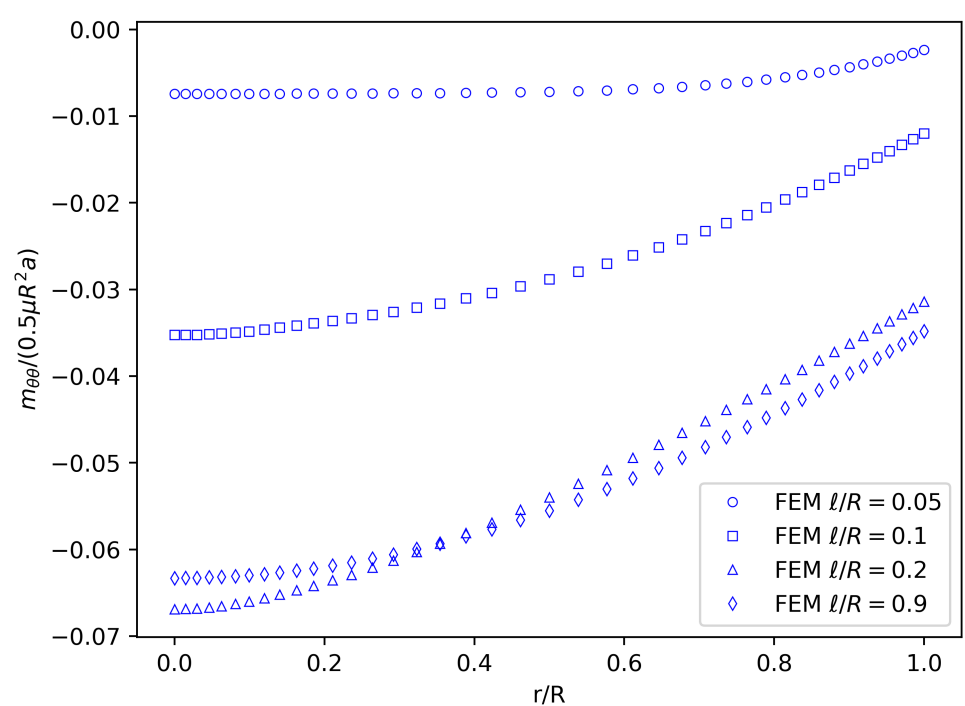

(b) Profiles of $m_{\theta \theta}$.

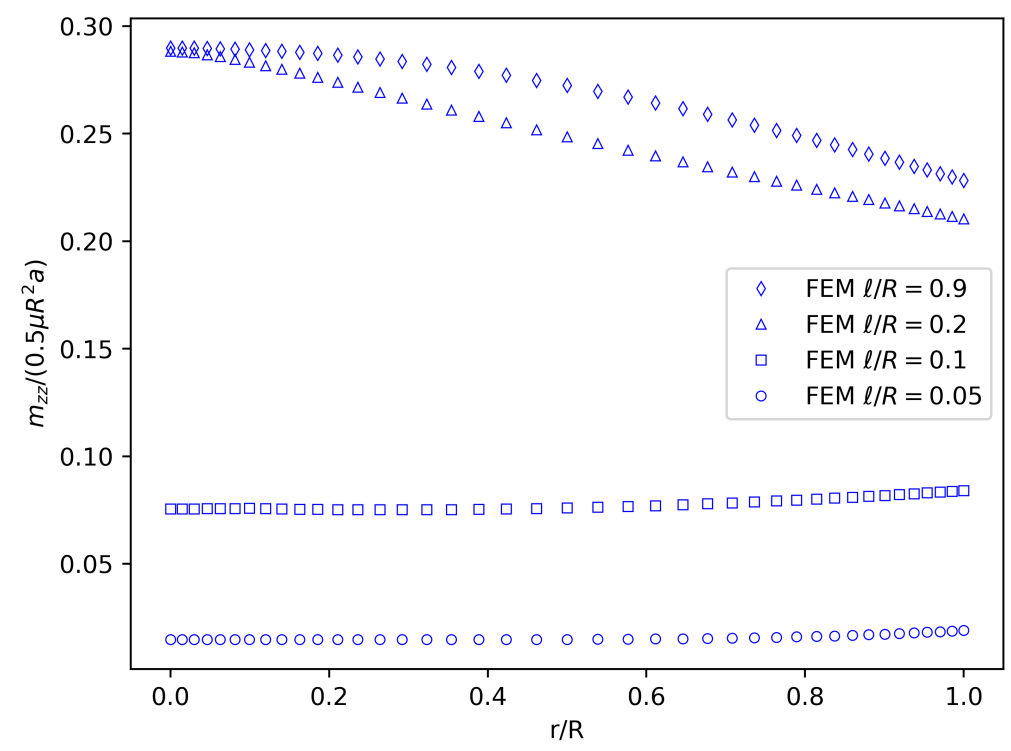

(c) Profiles of $m_{z z}$.

Fig. 19: Profiles of $m_{r r}, m_{\theta \theta}$ and $m_{z z}$ along a radius for various internal length values and $a=0.02 \mathrm{rad} / \mathrm{mm}$. The equivalent stress used in the yield function is given by equation (63). 


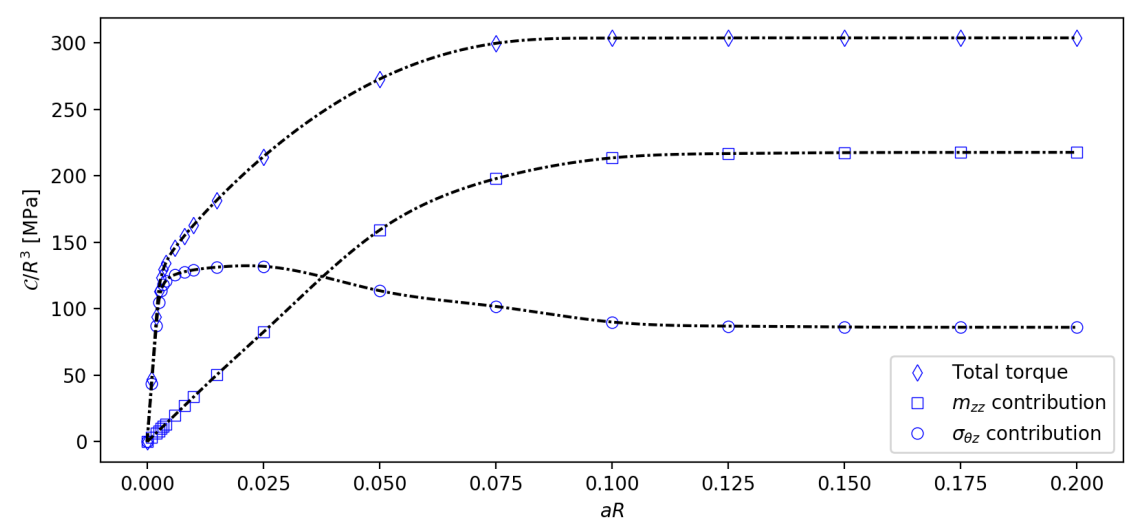

Fig. 20: Normalized torque as a function of the shear with $\ell / R=0.1$. Interpolated solutions are in black dash-dotted lines. The equivalent stress used in the yield function is given by equation (63).

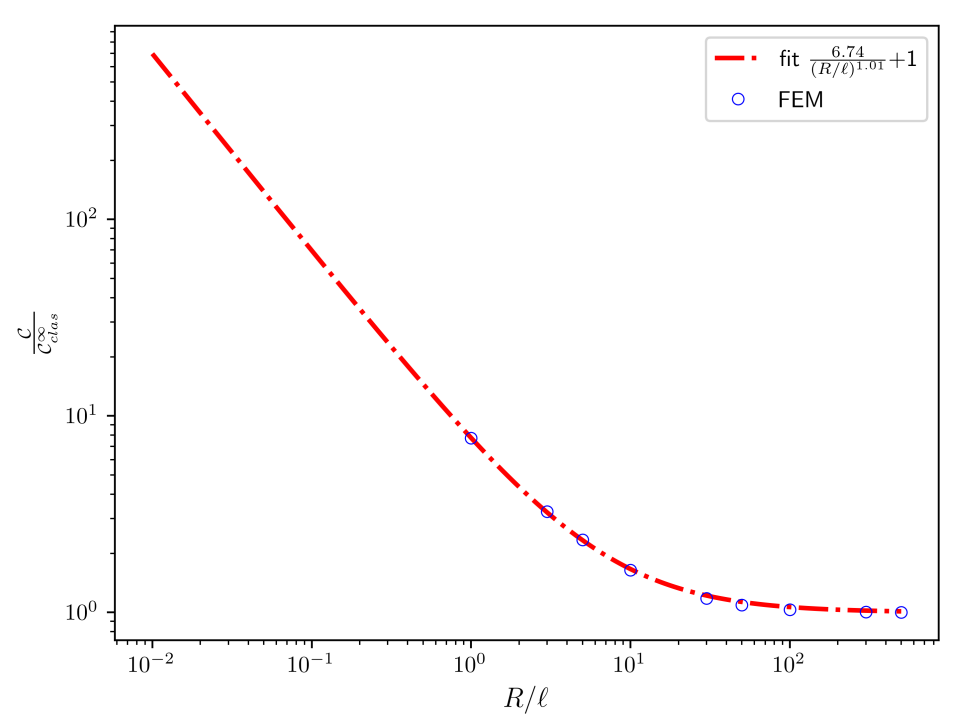

(a) Normalized torque as a function of the normalized radius for $\ell=0.1 \mathrm{~mm}$ and $a=0.02 \mathrm{rad} / \mathrm{mm}$.

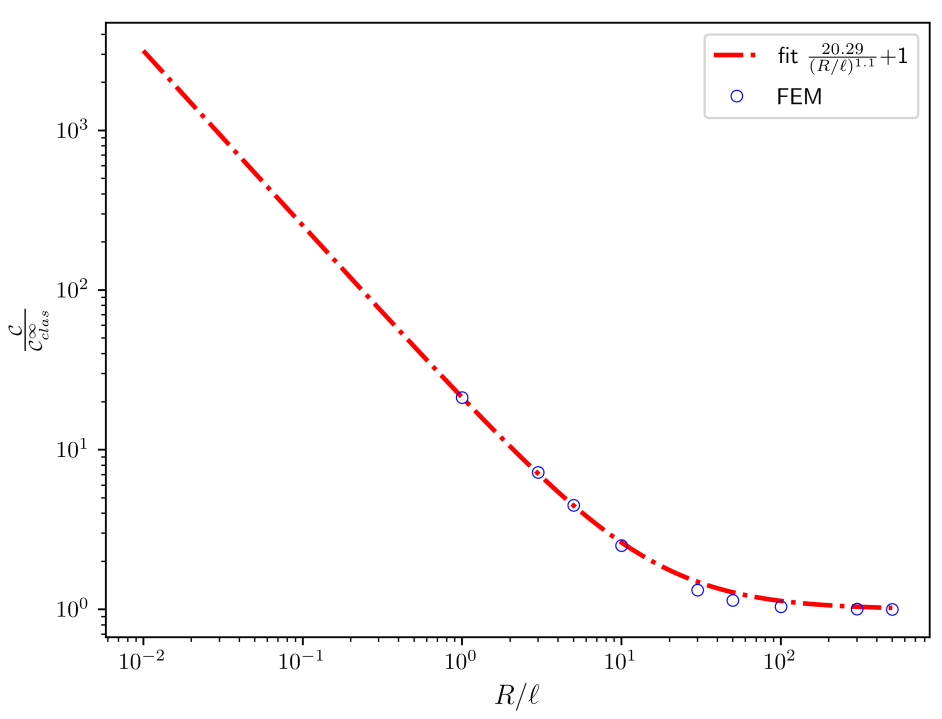

(b) Normalized torque as a function of the normalized radius for $\ell=0.1 \mathrm{~mm}$ and $a=0.2 \mathrm{rad} / \mathrm{mm}$.

Fig. 21: Torque normalized by the limit torque of a classical continuum as a function of the normalized radius for $\ell=0.1 \mathrm{~mm}$ and two values for $a$. The equivalent stress used in the yield function is given by equation (63). 


\section{Conclusion and outlook}

This work aimed at studying the torsion of isotropic, perfectly plastic Cosserat bars with circular cross-section. An analytical solution was derived in the case of a reduced form of the extended von Mises yield criterion only accounting for the symmetric part of the deviatoric stress tensor, though the stress tensor itself is non-symmetric in general. The torsional characteristic length given by equation (18) and derived in the elastic case, plays a major role on the resulting profiles of micro-rotation, stresses and couple-stresses. A size effect of the normalized torque proportional to $R^{-1}$ and dependent on the twist angle naturally arises in the torsional response of this model. Comparison with solutions obtained via the Finite Element Method by means of the software Zset shows perfect agreement.

A numerical investigation accounting for more general extended von Mises yield criteria shows comparable size effects, though the influence of the internal length on the resulting profiles can be very different. Depending on the yield criterion used, the different contributions to the total torque can display a saturation with respect to the twist angle.

Further numerical investigations of the response of Cosserat bars subjected to torsion should be performed in more general cases, including non-cylindrical bars [Drugan and Lakes, 2018], anisotropic elasticity [Taliercio and Veber, 2016], finite deformation as well as the incorporation of kinematic and isotropic hardening [Grammenoudis and Tsakmakis, 2009]. The main difficulty will consist in delineating the influence of each aspect of the model on the predicted size effects. Identification of material parameters with respect to experimental observation of size effects in torsion also remains to be done in the context of plasticity. The case of single crystalline bars will also be addressed since it combines plasticity, anisotropy and lattice curvature that can be captured by strain gradient plasticity models, see the recent work Scherer et al. [2020]. In the case of anisotropic materials like polycrystals, the question of plastic spin arises and its combination with curvature effects [Lachner et al., 1994; Bardella and Panteghini, 2015] with a significant amount of experimental results in torsion [Baczynski and Jonas, 1996]. Future work by the authors will focus on consequences of the torsion of Cosserat single crystal cylindrical bars to grain nucleation and application to recrystallization following the first steps by Ask et al. [2018, 2019].

\section{Acknowledgements}

This work is presented as a tribute to the memory of Prof. C.E. Eringen at the occasion of his 100th anniversary. SF thanks Prof. James D. Lee for the kind invitation to participate in this centennial volume. 


\section{References}

Ask, A., Forest, S., Appolaire, B. and Ammar, K. [2018] "Cosserat crystal plasticity with dislocation-driven grain boundary migration," Journal of Micromechanics and Molecular Physics 3, 1840009, doi:doi.org/10.1142/ S242491301840009X.

Ask, A., Forest, S., Appolaire, B. and Ammar, K. [2019] "A Cosserat-phase field theory of crystal plasticity and grain boundary migration at finite deformation," Continuum Mechanics and Thermodynamics 31, 1109-1141, doi: https://doi.org/10.1007/s00161-018-0727-6.

Baczynski, J. and Jonas, J. J. [1996] "Texture development during the torsion testing of $\alpha$-iron and two IF steels," Acta Materialia 44, 4273 - 4288, doi: 10.1016/1359-6454(96)00114-0.

Bardella, L. and Panteghini, A. [2015] "Modelling the torsion of thin metal wires by distortion gradient plasticity," Journal of the Mechanics and Physics of Solids 78, 467-492, doi:http://dx.doi.org/10.1016/j.jmps.2015.03.003.

Beheshti, A. [2018] "A numerical analysis of Saint-Venant torsion in strain-gradient bars," European Journal of Mechanics - A/Solids 70, 181-190, doi:10.1016/j. euromechsol.2018.02.001.

Besdo, D. [1974] "Ein beitrag zur nichtlinearen theorie des Cosserat-kontinuums," Acta Mechanica 20(1), 105-131.

Besson, J. and Foerch, R. [1997] "Large scale object-oriented finite element code design," Computer methods in applied mechanics and engineering 142(1-2), $165-187$.

Borst, R. d. [1993] "A generalization of $J_{2}$-flow theory for polar continua," Computer Methods in Applied Mechanics and Engineering 103, 347-362, doi:10.1016/ 0045-7825(93)90127-J.

Diepolder, W., Mannl, V. and Lippman, H. [1991] "The Cosserat continuum, a model for grain rotations in metals?" International journal of plasticity $\mathbf{7}(4)$, 313-328.

Dluzewski, P. [1992] "Finite elastic-plastic deformations of oriented media," in A. Benallal and D. Marquis (eds.), MECAMAT'g2, Int. Seminar on Multiaxial Plasticity (Cachan).

Drugan, W. and Lakes, R. [2018] "Torsion of a Cosserat elastic bar with square cross section: theory and experiment," Zeitschrift für angewandte Mathematik und Physik 69(2), 1-14.

Eringen, A. [1999] Microcontinuum field theories (Springer, New York).

Eringen, A. C. [1976] "Polar and nonlocal field theories," in A. C. Eringen (ed.), Continuum Physics. Vol 4. (Academic Press, London).

Fleck, N., Muller, G., Ashby, M. F. and Hutchinson, J. W. [1994] "Strain gradient plasticity: theory and experiment," Acta Metallurgica et materialia 42(2), 475-487.

Forest, S., Barbe, F. and Cailletaud, G. [2000] "Cosserat modelling of size effects in 
the mechanical behaviour of polycrystals and multiphase materials," International Journal of Solids and Structures 37, 7105-7126.

Forest, S. and Sievert, R. [2003] "Elastoviscoplastic constitutive frameworks for generalized continua," Acta Mechanica 160, 71-111.

Gauthier, R. and Jashman, W. [1975] "A quest for micropolar elastic constants," J. Appl. Mech. 42, 369-374.

Grammenoudis, P. and Tsakmakis, C. [2005] "Predictions of microtorsional experiments by micropolar plasticity," Proceedings of the Royal Society A: Mathematical, Physical and Engineering Sciences 461(2053), 189-205.

Grammenoudis, P. and Tsakmakis, C. [2009] "Isotropic hardening in micropolar plasticity," Archive of Applied Mechanics 79(4), 323-334.

Ieşan, D. [1971] "Torsion of micropolar elastic beams," International Journal of Engineering Science 9(11), 1047-1060.

Iesan, D. [2013] "Deformation of chiral rods in the strain gradient theory of thermoelasticity," European Journal of Mechanics - A/Solids 37, 351 - 360, doi: 10.1016/j.euromechsol.2012.08.006.

Kafadar, C. and Eringen, A. [1971] "Micropolar media: I the classical theory," Int. J. Engng Sci. 9, 271-305.

Kaiser, T., Forest, S. and Menzel, A. [2021] "A finite element implementation of the stress gradient theory," Meccanica 56, 1109-1128, doi:10.1007/ s11012-014-01266-3.

Kaplunov, J. and Lippmann, H. [1995] "Elastic-plastic torsion of a Cosserat-type rod," Acta mechanica 113(1), 53-62.

Kim, B. and Eringen, A. [1973] "Stress distribution around an elliptic hole in an infinite micropolar elastic plate," Letters in Applied and Engineering Sciences 1, 381-390.

Lachner, D., Lippmann, H. and Tóth, L. [1994] "On Cosserat plasticity and plastic spin for isotropic materials," Archives of Mechanics 46, 531-539.

Lakes, R. [1987] "Foam structures with a negative Poisson's ratio," Science 235, $1038-1040$.

Lazopoulos, K. and Lazopoulos, A. [2012] "On the torsion problem of strain gradient elastic bars," Mechanics Research Communications 45, 42-47, doi:10.1016/j. mechrescom.2012.06.007.

Lippmann, H. [1969] "Eine Cosserat-Theorie des plastischen Fließens," Acta Mechanica 8, 255-284.

Lippmann, H. [1995] "Cosserat plasticity and plastic spin," Appl. Mech. Rev. 48, $753-762$.

Neuber, H. [1966] "Ueber Probleme der Spannungskonzentration im CosseratKoerper," Acta Mechanica 2, 48-69, doi:10.1007/BF01176729.

Neuber, H. [1968] "On the effect of stress concentration in Cosserat continua," in E. Kröner (ed.), Mechanics of Generalized Continua (Springer Berlin Heidelberg, Berlin, Heidelberg), pp. 109-113, doi:10.1007/978-3-662-30257-6_13. 
Nowacki, W. [1986] Theory of asymmetric elasticity (Pergamon).

Onck, P. [2002] "Cosserat modeling of cellular solids," Comptes Rendus Mecanique 330, 717-722.

Reddy, G. V. K. and Venkatasubramanian, N. K. [1976] "Saint-Venant's problem for a micropolar elastic circular cylinder," International Journal of Engineering Science 14, 1047-1057, doi:10.1016/0020-7225(76)90099-9.

Sansour, C. [1998] "A theory of the elastic-viscoplastic Cosserat continuum," Archives of Mechanics 50, 577-597.

Sawczuk, A. [1967] "On yielding of Cosserat continua," Archiwum Mechaniki Stosowanej 19(3), 471.

Scherer, J. M., Phalke, V., Besson, J., Forest, S., Hure, J. and Tanguy, B. [2020] "Lagrange multiplier based vs micromorphic gradient-enhanced rate(in)dependent crystal plasticity modelling and simulation," Computer Methods in Applied Mechanics and Engineering 372, 113426, doi:10.1016/j.cma. 2020.113426.

Sievert, R. [1995] "Zur Zerlegung der Verformungsmaße des Cosserat-Kontinuums bei großen inelastischen Deformationen," Z. Angew. Math. Mech. 75, 205-206.

Smith, A. [1967] "Deformations of micropolar elastic solids," International Journal of Engineering Science 5(8), 637-651.

Taliercio, A. and Veber, D. [2016] "Torsion of elastic anisotropic micropolar cylindrical bars," European Journal of Mechanics-A/Solids 55, 45-56.

Toupin, R. [1962] "Elastic materials with couple stresses," Arch. Rat. Mech. Anal. 11, 385-414.

Yang, J. and Lakes, R. [1981] "Transient study of couple stress effects in compact bone : torsion," J. of Biomech. Engng 103, 275-279. 